\title{
Detection by Time Reversal: Single Antenna
}

\author{
José M. F. Moura, Fellow, IEEE, and Yuanwei Jin, Member, IEEE
}

\begin{abstract}
This paper studies the binary hypothesis test of detecting the presence or absence of a target in a highly cluttered environment by using time reversal. In time reversal, the backscatter of a signal transmitted into a scattering environment is recorded, delayed, energy normalized, and retransmitted through the medium. We consider two versions of the test-target channel frequency response assumed known or unknown-and, for each version, contrast two approaches: conventional detection (where no time reversal occurs) and time reversal detection. This leads to four alternative formulations for which we derive the optimal detector and the generalized likelihood ratio test, when the target channel frequency response is known or unknown, respectively. We derive analytical expressions for the error probabilities and the threshold for all detectors, with the exception of the time reversal generalized likelihood ratio test. Experiments with real-world electromagnetic data for two channels (free space with a target immersed in 20 scatterers and a duct channel) confirm the analytical results and show that time reversal detection provides significant gains over conventional detection. This gain is explained by the empirical distribution or type of the target channel frequency response-richer scattering channels induce types with heavier tails and larger time reversal detection gains.
\end{abstract}

Index Terms-Adaptive waveform, detection, empirical distribution, matched filter, time reversal, type, waveform reshape.

\section{INTRODUCTION}

C HANNEL multipath significantly affects the performance of traditional detectors, e.g., the matched filter. Usually, multipath is thought to be detrimental and a negative whose effects should be minimized. Time reversal presents the opposite opportunity-multipath as a positive, the more the better. In time reversal signal processing, a signal is first radiated through a rich scattering medium. The backscattered signal is then recorded, delayed, time reversed, energy normalized, and retransmitted. The technique of time reversal is not new, but a thorough theory of detection for this setting is lacking. This paper addresses this gap. We study time reversal detection of a target immersed in a rich scattering environment. We focus on determining the performance gain, if any, provided by the time reversal based detector over conventional detection techniques. We carry out the following plan.

1) Formulate a time reversal approach to detection and contrast it with the conventional approach.

2) Derive the detectors for each of these approaches.

Manuscript received July 15, 2005; accepted March 21, 2006. This work was supported by the Mathematical Time Reversal Methods Program, DSO-CMP, Defence Advanced Research Projects Agency through the Army Research Office under Grant W911NF-04-1-0031.The associate editor coordinating the review of this manuscript and approving it for publication was Prof. Mats Viberg.

The authors are with the Department of Electrical and Computer Engineering, Carnegie-Mellon University, Pittsburgh, PA 15213 USA (e-mail: moura@ece. cmu.edu; ywjin@ece.cmu.edu).

Color versions of Figs. 1 and 3-9 are available online at http://ieeexplore. ieee.org.

Digital Object Identifier 10.1109/TSP.2006.882114
3) Detail the performance of the detectors analytically and experimentally.

4) Test the detectors with real electromagnetic (EM) data collected with two different laboratory experiments.

Our results are conclusive.

1) Time reversal detection provides significant gains over conventional detection.

2) The time reversal detection gain is verified experimentally for the first time with electromagnetic real-world experiments.

3) The time reversal detection gain is directly related to the type $e^{1}$ of the target channel frequency response- the gain is larger for heavy tailed channel types.

4) The time reversal detection gain arises because the transmitter reshapes the waveform to best match the channel.

\section{A. On Time Reversal}

Time reversal (TR), known in optics as phase conjugation, has been used to increase resolution by exploiting scattering and multipath in inhomogeneous channels. Fink and collaborators have published extensively on time reversal in acoustics and ultrasound [2]-[6]. These works demonstrated superresolution focusing in the ultrasound domain. In their work, an ultrasound source is placed in a water tank with a large number of scatterers. The scattered acoustic signal is recorded by an array of sensors and retransmitted through the same medium after being time reversed. Their experiments demonstrate that the acoustic energy refocus at the source with much higher resolution than predicted by the Rayleigh resolution limit, i.e., they demonstrate superresolution focusing. More recently, large-scale acoustics experiments in the ocean confirmed the resolution ability of time reversal in real acoustic propagation environments [7], [8]. There is a growing literature on time reversal in these acoustic and ultrasound fields, as well as on studies of time reversal in random environments [9] and in several applications domains, including imaging [10], [11] or communications [12]-[14]. Focusing in the electromagnetic domain has recently been demonstrated in [15] and [16]. In [17], we presented a TR-based interference canceller to mitigate the effect of clutter in the electromagnetic domain. None of these works has studied the problem of detection using time reversal, derived the detectors, and studied time reversal detection analytically and by experimentation with real electromagnetic data. This is what this paper pursues and accomplishes. To stress the focus on the impact of time reversal, we consider the detection of a target in clutter with a single antenna. This precludes the use of narrow-band multiple signal classification and subspace type algorithms where the number of clutter returns is restricted to be smaller than the number of array elements.

\footnotetext{
${ }^{1}$ The expression type is used in its information theoretic sense of empirical distribution [1].
} 
The remainder of this paper is organized as follows. In Section II, we describe the time reversal measurement protocol and present the statistics of the measurements. Section III formalizes the single binary hypothesis test problem with a single receiving antenna under study - target present or absent in high clutter, the two approaches-conventional and time reversal - that we consider, and their two versions-ideal and realistic-where the target channel response is known or unknown, respectively. The section presents the optimal detectors and the generalized likelihood ratio tests for the ideal and realistic versions of each approach. The section derives analytical expressions for the thresholds and error probabilities for each detector, with the exception of the time reversal generalized likelihood ratio test. Section IV derives an expression for the detection gain provided by time reversal detection over conventional detection in the ideal case of known target channel response. Section V tests all detectors in real-world scenarios with electromagnetic data. The section presents experiments with two channels (free space with many scatterers and a duct channel) that confirm the analytical results and show that time reversal delivers significant detection gains. The section illustrates how these detection gains relate to the empirical distribution or type of the target channel frequency response. We summarize our results in Section VI.

\section{B. Notation}

Lower case boldface letters denote vectors and upper case boldface letters denote matrices; $(\cdot)^{*}$ stands for conjugate, $(\cdot)^{T}$ for transpose, and $(\cdot)^{H}$ for Hermitian transpose; $\mathfrak{R}(A)$ and $\mathfrak{I}(A)$ are the real and the imaginary parts of $A$, respectively; $\mathbf{x} \odot \mathbf{y}$ is the Hadamard product or component-wise product of two vectors or two matrices (with the same dimensions), while $\mathbf{A} \otimes \mathbf{B}$ is the Kronecker product of $\mathbf{A}$ and $\mathbf{B} ; \mathrm{E}\{\cdot\}$ is the expected value of a random quantity; $\mathbf{I}_{m}$ is the identity matrix of order $m$; $\operatorname{vec}\{\mathbf{Y}\}$ stands for the column vector that results when we stack the columns of the matrix $\mathbf{Y}$ and $\operatorname{diag}\{\mathbf{x}\}$ is a diagonal matrix whose diagonal is the vector $\mathbf{x} ;\|\cdot\|$ is the vector or matrix Frobenius norm; finally, we recall that the probability density function of the $Q$-dimensional complex circular Gaussian random vector $\mathbf{y}$ with mean $\overline{\mathbf{y}}$ and covariance $\Sigma_{\mathbf{y}}$, e.g., [18], is

$$
p_{\mathbf{y}}(\mathbf{y})=\mathcal{C N}\left(\overline{\mathbf{y}}, \Sigma_{\mathbf{y}}\right)=\frac{1}{\pi^{Q}\left|\Sigma_{\mathbf{y}}\right|} e^{-[\mathbf{y}-\overline{\mathbf{y}}]^{H} \Sigma_{\mathbf{y}}^{-1}[\mathbf{y}-\overline{\mathbf{y}}]} .
$$

When the vector is white, $\Sigma_{\mathbf{y}}=\sigma_{\mathbf{y}}^{2} \mathbf{I}$, and $\sigma_{\mathbf{y}}^{2}$ is referred to as the variance of the random vector.

\section{Time Reversal Measurements}

We consider an active radar (or sonar) system with a single receiving antenna. The transmitted signal $s(t)$ is a wide-band signal with duration $2 T$ and bandwidth $B=(2 \pi / T)$. Its discrete Fourier transform is $S\left(\omega_{q}\right), \omega_{q}=(2 \pi / T(Q-1))\left(q_{1}+\right.$ $q), q=0,1, \ldots, Q-1$, and $q_{1}$ is a constant. For real-valued time-dependent signals $s(t)$, the discrete Fourier transform of its time reversed version $s\left(T_{c}-t\right)$, where $T_{c}$ is a sufficiently long delay, is simply given by $e^{-j \omega_{q} T_{c}} S^{*}\left(\omega_{q}\right)$; in other words, besides a phase shift, time reversal becomes phase conjugation in the frequency domain (see, e.g., [19]).
This paper studies the impact of time reversal in target detection in cluttered environments. We assume that we have independent measurements of the clutter when no target is present and that the clutter remains stationary. To emphasize the impact of the channel propagation effects (multipath) induced by the clutter and to keep the focus on the role of time reversal on detection, we consider in this paper the extreme case of either a single antenna in the monostatic context or a single transmitting antenna and a single receiving antenna in the bistatic problem.

We introduce two frequency responses: 1) the clutter frequency response $H_{c}\left(\omega_{q}\right), q=0, \ldots, Q-1$, which is the response of the clutter when no target is present; and 2) the target channel frequency response $H_{t}\left(\omega_{q}\right), q=0, \ldots, Q-1$, which is the difference between the channel response when a target is present and the channel response when no target is present. As such, $H_{t}\left(\omega_{q}\right)$ represents all the changes to $H_{c}\left(\omega_{q}\right)$ induced by the presence of the target and, in particular, includes secondary backscatter, i.e., backscatter from the clutter to the target that is then radiated back to the receiving antenna.

The problem we consider is the following. We assume that there is an initial phase where the clutter frequency response $H_{c}\left(\omega_{q}\right)$ can be learned. Then, the deterministic part ${ }^{2}$ of the response that can be computed by propagating the transmitted signals through $H_{c}\left(\omega_{q}\right)$ will be subtracted out and we work with the resulting signals. We call this background subtraction. We explain this next.

\section{A. Clutter Response}

This phase learns the clutter response $H_{c}\left(\omega_{q}\right)$. Assume that no target is present. The single antenna probes the channel with the wide-band signal $S\left(\omega_{q}\right), q=0, \ldots, Q-1$, with energy

$$
E_{s}=\frac{1}{Q} \sum_{q=0}^{Q-1}\left|S\left(\omega_{q}\right)\right|^{2} .
$$

We repeat the probing to obtain $L$ independent snapshots $Y_{\mathrm{cl}}\left(\omega_{q}\right)$ where

$$
\begin{aligned}
Y_{\mathrm{cl}}\left(\omega_{q}\right)=S\left(\omega_{q}\right) H_{c}\left(\omega_{q}\right) & +U_{l}\left(\omega_{q}\right) \\
q & =0, \ldots, Q-1, l=1, \ldots, L .
\end{aligned}
$$

In (3), $U_{l}\left(\omega_{q}\right)$ is additive, zero mean, circular complex white Gaussian noise with diagonal covariance $\sigma_{u}^{2} \mathbf{I}$. The minimum mean square error estimate of the clutter response is

$$
\begin{aligned}
\hat{H}_{c}\left(\omega_{q}\right) & =S^{-1}\left(\omega_{q}\right) \frac{1}{L} \sum_{l=1}^{L} Y_{\mathrm{cl}}\left(\omega_{q}\right) \\
& =H_{c}\left(\omega_{q}\right)+S^{-1}\left(\omega_{q}\right) \frac{1}{L} \sum_{l=1}^{L} U_{l}\left(\omega_{q}\right) .
\end{aligned}
$$

For $L$ sufficiently large, the clutter response is well estimated from the $L$ probing snapshots, i.e.,

$$
\hat{H}_{c}\left(\omega_{q}\right) \approx H_{c}\left(\omega_{q}\right)
$$

so, we safely assume in the sequel that $H_{c}\left(\omega_{q}\right)$ is accurately known.

\footnotetext{
${ }^{2}$ This assumption may not be applicable in many radar/sonar environments where the scattering characteristics must be described stochastically.
} 


\section{B. Clutter Suppression-Background Subtraction}

Because the clutter response is assumed known, we can suppress the clutter by simple background subtraction. Background subtraction is widely used in many applications from radar to image or video processing. Assume that the backscatter of the channel when probed by a signal $S\left(\omega_{q}\right)$ is $R\left(\omega_{q}\right)$. Part of this signal is the backscatter from the clutter. The clutter suppressed signal is then

$$
Y\left(\omega_{q}\right)=R\left(\omega_{q}\right)-S\left(\omega_{q}\right) H_{c}\left(\omega_{q}\right) .
$$

We will formulate the detection problems that we study in this paper in terms of the residual signals $Y\left(\omega_{q}\right)$ rather than the signals $R\left(\omega_{q}\right)$.

\section{Time Reversal: Measurement Protocol}

We assume that the clutter response has been learned as explained in (4). The second phase monitors the channel. The monitoring protocol in Section III when we use time reversal is in two steps, which are repeated $M$ times to obtain $M$ snapshots.

1) Probing: This step transmits at the $m$ th snapshot, the signal $S\left(\omega_{q}\right), q=0, \ldots, Q-1$. When a target is present, the channel backscattered signal received by the antenna is

$$
\begin{array}{r}
R_{m}\left(\omega_{q}\right)=S\left(\omega_{q}\right)\left[H_{t}\left(\omega_{q}\right)+H_{c}\left(\omega_{q}\right)\right]+V_{m}\left(\omega_{q}\right) \\
q=0, \ldots, Q-1, \quad m=1, \ldots, M
\end{array}
$$

where $V_{m}\left(\omega_{q}\right)$ is additive, zero mean, circular complex white Gaussian noise, with diagonal covariance $\sigma_{v}^{2} \mathbf{I}$. In (7), $H_{t}\left(\omega_{q}\right)$ is the target channel response, which, as explained above, is the difference between the channel response when clutter and target are present and when only clutter is present. By background subtraction [see (6)], the clutter suppressed signal is

$$
\begin{aligned}
Y_{m}\left(\omega_{q}\right) & =R_{m}\left(\omega_{q}\right)-S\left(\omega_{q}\right) H_{c}\left(\omega_{q}\right) \\
& =S\left(\omega_{q}\right) H_{t}\left(\omega_{q}\right)+V_{m}\left(\omega_{q}\right), \\
& q=0, \ldots, Q-1, \quad m=1, \ldots M .
\end{aligned}
$$

2) Time Reversal: In this step, we use time reversal, ${ }^{3}$ which, as observed before, corresponds to phase conjugation in the frequency domain. Time reversing the clutter suppressed received signal in (8), we obtain

$$
\begin{aligned}
& Y_{m}^{*}\left(\omega_{q}\right)=S^{*}\left(\omega_{q}\right) H_{t}^{*}\left(\omega_{q}\right)+V_{m}\left(\omega_{q}\right), \\
& \quad q=0, \ldots, Q-1, \quad m=1, \ldots M .
\end{aligned}
$$

Next, the signal $Y_{m}^{*}\left(\omega_{q}\right)$ is normalized to the energy $E_{s}$ of the original signal $S\left(\omega_{q}\right), q=0, \ldots, Q-1$ by an energy normalization factor $k_{m}$

$$
k_{m}=\sqrt{\frac{Q E_{s}}{\sum_{q=0}^{Q-1}\left|Y_{m}\left(\omega_{q}\right)\right|^{2}}} .
$$

Note that the energy normalization factor $k_{m}$ changes from snapshot to snapshot but is known since it is computed from the received data $Y_{m}\left(\omega_{q}\right), q=0, \ldots, Q-1$.
The received signal is for all $q=0, \ldots, Q-1, \quad m=$ $1, \ldots, M$ :

$$
R_{m}^{d}\left(\omega_{q}\right)=k_{m} Y_{m}^{*}\left(\omega_{q}\right)\left[H_{t}\left(\omega_{q}\right)+H_{c}\left(\omega_{q}\right)\right]+W_{m}\left(\omega_{q}\right) .
$$

If no target is present, $H_{t}\left(\omega_{q}\right)=0$ in (11). The term $W_{m}\left(\omega_{q}\right), q=0, \ldots, Q-1, m=1, \ldots, M$, is a circular complex zero mean white Gaussian noise with variance $\sigma_{w}^{2}$.

As in (8), the known component $k_{m} Y_{m}^{*}\left(\omega_{q}\right) H_{c}\left(\omega_{q}\right)$ from the backscattered signal received by the antenna is subtracted out. The resulting signal is, $\forall q=0, \ldots, Q-1, m=1, \ldots, M$

$$
\begin{aligned}
X_{m}\left(\omega_{q}\right)= & R_{m}^{d}\left(\omega_{q}\right)-k_{m} Y_{m}^{*}\left(\omega_{q}\right) H_{c}\left(\omega_{q}\right) \\
= & k_{m}\left[S\left(\omega_{q}\right) H_{t}\left(\omega_{q}\right)+V_{m}\left(\omega_{q}\right)\right]^{*} H_{t}\left(\omega_{q}\right) \\
& +W_{m}\left(\omega_{q}\right) \\
= & k_{m} S^{*}\left(\omega_{q}\right)\left|H_{t}\left(\omega_{q}\right)\right|^{2} \\
& +k_{m} V_{m}^{*}\left(\omega_{q}\right) H_{t}\left(\omega_{q}\right)+W_{m}\left(\omega_{q}\right) .
\end{aligned}
$$

The setup just described assumes that the clutter remains static or invariant so that the simple background subtraction in (8) and (12) effectively suppresses the clutter response.

For detection by time reversal, we have both the direct signals $Y_{m}\left(\omega_{q}\right)$ in (8) and the time reversal signals $X_{m}\left(\omega_{q}\right)$ in (13), $q=0, \ldots, Q-1$, and $m=1, \ldots, M$.

\section{Time Reversal Measurements: Vector Notation}

Before we state formally the hypothesis testing problem, we express the time reversal measurements in vector notation. We collect for each snapshot $m$ the frequency responses $Y_{m}\left(\omega_{q}\right)$ in a $Q$-dimensional vector $\mathbf{y}_{m}$ and then stack these vectors in the $Q M$-dimensional vector $\mathbf{y}$, i.e.,

$$
\begin{aligned}
\mathbf{y}_{m} & =\left[Y_{m}\left(\omega_{0}\right) \cdots Y_{m}\left(\omega_{Q-1}\right)\right]^{T} \\
\mathbf{y} & =\operatorname{vec}\left\{\left[\mathbf{y}_{1}, \ldots, \mathbf{y}_{M}\right]\right\} .
\end{aligned}
$$

Similarly, the $Q$-dimensional vectors $\mathbf{s}, \mathbf{x}_{m}, \mathbf{h}_{t}, \mathbf{v}_{m}$, and $\mathbf{w}_{m}$ collect the spectrum of the transmitted signal $S\left(\omega_{q}\right)$, the signals $X_{m}\left(\omega_{q}\right)$ in (13), the target channel frequency response $H_{t}\left(\omega_{q}\right)$, and the noises $V_{m}\left(\omega_{q}\right)$ and $W_{m}\left(\omega_{q}\right)$. The vectors $\mathbf{x}_{m}, \mathbf{v}_{m}$, and $\mathbf{w}_{m}$ are then stacked in the $Q M$-dimensional vectors $\mathbf{x}, \mathbf{v}$, and $\mathbf{w}$, respectively. Finally, we introduce

$$
\begin{aligned}
\mathbf{S} & =\operatorname{diag}\{\mathbf{s}\} \\
\mathbf{z}_{m} & =\left[\begin{array}{ll}
\mathbf{y}_{m}^{H} & \mathbf{x}_{m}^{T}
\end{array}\right]^{T} \\
\mathbf{1} & =\left[\begin{array}{ll}
1 \cdots 1 & \cdots \\
\mathbf{k} & =\left[k_{1}, \ldots, k_{M}\right.
\end{array}\right]^{T} \\
\mathbf{K} & =\operatorname{diag}\{\mathbf{k}\} .
\end{aligned}
$$

The vector $\mathbf{k}$ vectorizes the energy normalization gains, while the diagonal matrix $\mathbf{K}$ has these gains in the diagonal. The $M$-dimensional vector $\mathbf{1}$ is a vector of ones. The $2 Q$-dimensional vector $\mathbf{z}_{m}$ vectorizes all the $Y_{m}^{*}\left(\omega_{q}\right)$ and $X_{m}\left(\omega_{q}\right)$ data for snapshot $m$. However, we use a slightly different notation for the $2 Q M$-dimensional vector $\mathbf{z}$. This vector

$$
\mathbf{z}=\left[\begin{array}{ll}
\mathbf{y}^{H} & \mathbf{x}^{T}
\end{array}\right]^{T}
$$


concatenates the vectors $\mathbf{y}^{*}$ and $\mathbf{x}$ rather than simply stacking the vectors $\mathbf{z}_{m}$. The vector $\mathbf{z}$ stacks the data for all the $M$ snapshots. We emphasize that in $\mathbf{z}_{m}$ and $\mathbf{z}$, we stack the time reversed, i.e., the conjugates, $\mathbf{y}_{m}^{*}$ and $\mathbf{y}^{*}$ with $\mathbf{x}_{m}$ and $\mathbf{x}$, respectively.

We now use these vectors to write compactly the signals at the different phases of the time reversal measurement protocol, using the Hadamard $\odot$ product introduced in Section I. We have

$$
\begin{aligned}
\mathbf{y}_{m}= & {\left[S\left(\omega_{q}\right) H_{t}\left(\omega_{q}\right)+V_{m}\left(\omega_{q}\right)\right] } \\
= & \mathbf{S h}_{t}+\mathbf{v}_{m}, \quad m=1, \ldots, M \\
\mathbf{x}_{m}= & {\left[k_{m} S^{*}\left(\omega_{q}\right)\left|H_{t}\left(\omega_{q}\right)\right|^{2}\right.} \\
& \left.+k_{m} H_{t}\left(\omega_{q}\right) V_{m}^{*}\left(\omega_{q}\right)+W_{m}\left(\omega_{q}\right)\right] \\
= & k_{m} \mathbf{y}_{m}^{*} \odot \mathbf{h}_{t}+\mathbf{w}_{m} \\
= & k_{m} \mathbf{S}^{*} \mathbf{h}_{t}^{*} \odot \mathbf{h}_{t}+k_{m} \mathbf{v}_{m}^{*} \odot \mathbf{h}_{t}+\mathbf{w}_{m}, \\
& m=1, \ldots, M
\end{aligned}
$$

where in (22) and (24), we indicate explicitly the entries of $\mathbf{y}_{m}$ and $\mathbf{x}_{m}$, respectively. Equations (23)-(26) assume a target is present. If no target is present, then $\mathbf{h}_{t}=\mathbf{0}$, and the received data $\mathbf{y}_{m}$ and $\mathbf{x}_{m}$ are simply the noises $\mathbf{v}_{m}$ and $\mathbf{w}_{m}$, respectively.

Remark: In the setup described in Section II-A, we collect a total of $2 M$ data snapshots, i.e., $M$ snapshots of $\mathbf{y}_{m}$ and $M$ snapshots of $\mathbf{x}_{m}$, where each $\mathbf{x}_{m}$ is obtained by transmitting the corresponding time reversed signal $\mathbf{y}_{m}$. In practice, other transmission strategies may be adopted while keeping the total number of data snapshots unchanged. For instance, we can transmit a single $M_{Y}=1$ snapshot of $\mathbf{y}_{m}$ and $M_{X}=2 M-1$ snapshots of $\mathbf{x}_{m}$, keeping $M_{Y}+M_{X}=2 M$. It is anticipated that the performance of time reversal detection will vary with different transmission strategies. In this paper, we use the simple strategy where we alternate each $\mathbf{y}_{m}$ transmission with an $\mathbf{x}_{m}$ transmission, i.e., $M_{X}=M_{Y}=M$.

\section{E. Noise and Data Statistics}

Finally, to complete the model, we summarize the statistics assumed. The noise vector $\mathbf{v}_{m}$ is a circular complex Gaussian random vector, i.e.,

$$
\mathbf{v}_{m} \sim \mathcal{C N}\left(0, \sigma_{v}^{2} \mathbf{I}_{Q}\right)
$$

See (1) for the notation used and the explicit expression for the probability density function. The real and imaginary components of $\mathbf{v}_{m}$ are, respectively, $\mathfrak{R}\left\{\mathbf{v}_{m}\right\} \sim \mathcal{N}\left(0,\left(\sigma_{v}^{2} / 2\right) \mathbf{I}_{Q}\right)$ and $\mathfrak{I}\left\{\mathbf{v}_{m}\right\} \sim \mathcal{N}\left(0,\left(\sigma_{v}^{2} / 2\right) \mathbf{I}_{Q}\right)$, e.g., [20]. Similarly, the noise vector $\mathbf{w}_{m}$ is the complex Gaussian random vector

$$
\mathbf{w}_{m} \sim \mathcal{C N}\left(0, \sigma_{w}^{2} \mathbf{I}_{Q}\right)
$$

The noises $\mathbf{v}_{m}$ and $\mathbf{w}_{m}$ are uncorrelated and independent of the transmitted signal.

We now consider the statistics of the data $\mathbf{y}, \mathbf{x}$, and $\mathbf{z}$. When no target is present, $\mathbf{h}_{t}=\mathbf{0}$, and it is straightforward to derive from the statistics of $\mathbf{v}_{m}$ and $\mathbf{w}_{m}$ that

$$
\begin{aligned}
& \mathbf{y} \sim \mathcal{C N}\left(\mathbf{0}, \sigma_{v}^{2} \mathbf{I}_{M} \otimes \mathbf{I}_{Q}\right) \\
& \mathbf{x} \sim \mathcal{C} \mathcal{N}\left(\mathbf{0}, \sigma_{w}^{2} \mathbf{I}_{M} \otimes \mathbf{I}_{Q}\right)
\end{aligned}
$$

where $\otimes$ is the tensor product introduced in Section I. We explicitly indicate $\mathbf{I}_{M} \otimes \mathbf{I}_{Q}$ to emphasize that the vectors $\mathbf{y}$ and $\mathbf{x}$ are the result of stacking $M$ vectors of dimension $Q$. From (29) and (30), and noting further that, when no target is present, $\mathbf{y}^{*}$ and $\mathbf{x}$ are statistically independent, the probability density function of $\mathbf{z}$, denoted by $p\left(\mathbf{z} \mid \mathbb{H}_{0}\right)$, is given by

$$
p\left(\mathbf{z} \mid \mathbb{H}_{0}\right)=\mathcal{C N}\left(\left[\begin{array}{l}
\mathbf{0} \\
\mathbf{0}
\end{array}\right],\left[\begin{array}{cc}
\sigma_{v}^{2} \mathbf{I}_{M} \otimes \mathbf{I}_{Q} & \mathbf{0} \\
\mathbf{0} & \sigma_{w}^{2} \mathbf{I}_{M} \otimes \mathbf{I}_{Q}
\end{array}\right]\right) .
$$

When a target is present, the statistics of $\mathbf{y}$ are still straightforward

$$
\begin{aligned}
Y_{m}\left(\omega_{q}\right) & \sim \mathcal{C N}\left(S\left(\omega_{q}\right) H_{t}\left(\omega_{q}\right), \sigma_{v}^{2}\right) \\
\mathbf{y} & \sim \mathcal{C N}\left(\mathbf{y}_{t}=\mathbf{1} \otimes \mathbf{S h}_{t}, \sigma_{v}^{2} \mathbf{I}_{M} \otimes \mathbf{I}_{Q}\right)
\end{aligned}
$$

however, the statistics of $\mathbf{x}$, under the time reversal protocol, are more complicated due to the energy normalization factors $k_{m}$. We indicate the conditional statistics of $\mathbf{x}$ given the gains $k_{m} \mathrm{~s}$. Then, conditioned on the vector of energy normalization factors k, see (19)

$$
\begin{aligned}
X_{m}\left(\omega_{q}\right) \sim & \mathcal{C N}\left(k_{m} S\left(\omega_{q}\right)\left|H_{t}\left(\omega_{q}\right)\right|^{2},\right. \\
& \left.\sigma_{v}^{2} k_{m}^{2}\left|S\left(\omega_{q}\right)\right|^{2}\left|H_{t}\left(\omega_{q}\right)\right|^{2}+\sigma_{w}^{2}\right) \\
\mathbf{x} \sim & \mathcal{C N}\left(\mathbf{x}_{t}=\mathbf{k} \otimes\left[\mathbf{S}^{*} \mathbf{h}_{t}^{*} \odot \mathbf{h}_{t}\right]\right. \\
& \left.\sigma_{v}^{2} \mathbf{K}^{2} \otimes \operatorname{diag}\left\{\mathbf{h}_{t}^{*} \odot \mathbf{h}_{t}\right\}+\sigma_{w}^{2} \mathbf{I}_{M} \otimes \mathbf{I}_{Q}\right)
\end{aligned}
$$

where $\mathbf{K}$ is the diagonal matrix of normalization factors defined in (20). To get the statistics of $\mathbf{z}$, we need to consider the cross-statistics of $\mathbf{y}^{*}$ and $\mathbf{x}$ conditioned on all $k_{m}$; we will not provide details here. After some manipulations, we find that the probability density function of $\mathbf{z}$ when a target is present, denoted by $p\left(\mathbf{z} \mid \mathbb{H}_{1}\right)$, is [see (36) at the bottom of the page].

\section{Time ReVERSAl Detection: Single Antenna}

We consider now the hypothesis test of detecting a target buried in a rich cluttered environment with a single antenna. Under the null hypothesis $\mathbb{H}_{0}$, the data are target signal free, while under the alternative hypothesis $\mathbb{H}_{1}$, the measured data

$$
p\left(\mathbf{z} \mid \mathbb{H}_{1}\right)=\mathcal{C N}\left(\left[\begin{array}{c}
1 \otimes \mathbf{S}^{*} \mathbf{h}_{t}^{*} \\
\mathbf{k} \otimes\left[\mathbf{S}^{*} \mathbf{h}_{t}^{*} \odot \mathbf{h}_{t}\right]
\end{array}\right],\left[\begin{array}{cc}
\sigma_{v}^{2} \mathbf{I}_{M} \otimes \mathbf{I}_{Q} & \sigma_{v}^{2} \mathbf{K} \otimes \operatorname{diag}\left\{\mathbf{h}_{t}^{*}\right\} \\
\sigma_{v}^{2} \mathbf{K} \otimes \operatorname{diag}\left\{\mathbf{h}_{t}\right\} & \sigma_{v}^{2} \mathbf{K}^{2} \otimes \operatorname{diag}\left\{\mathbf{h}_{t}^{*} \odot \mathbf{h}_{t}\right\}+\sigma_{w}^{2} \mathbf{I}_{M} \otimes \mathbf{I}_{Q}
\end{array}\right]\right) .
$$


contains a target signal. We start by detailing in Section III-A the detection problems we consider. In the remaining parts of this section we describe the detectors and their error performance.

\section{A. Detection Problems}

Under the measurement protocols described in the previous section, we first learn the clutter and then use background subtraction. This allows us to derive a simpler equivalent detection problem where, under $\mathbb{H}_{1}$, the measured data, after canceling the effect of the clutter, are equivalent to the signals $Y_{m}\left(\omega_{q}\right)$ and $X_{m}\left(\omega_{q}\right), q=0, \ldots, Q-1, m=1, \ldots, M$, given by (8) and (13), or are equivalently described by (23) and (26). For detection purposes, we can then ignore the role of the clutter response $H_{c}\left(\omega_{q}\right)$ and assume the equivalent signal model $Y_{m}\left(\omega_{q}\right)$ and $X_{m}\left(\omega_{q}\right), q=0, \ldots, Q-1, m=1, \ldots, M$, where only the effective target channel response $H_{t}\left(\omega_{q}\right)$ is explicit.

1) Ideal and Realistic Scenarios: For this detection problem, we consider two different versions. In the first one, which we refer to as the ideal scenario, the target channel response $H_{t}\left(\omega_{q}\right)$ or, in vector form, $\mathbf{h}_{t}$, is assumed known. In the other version, termed realistic, the target channel response $\mathbf{h}_{t}$ is assumed not known. Although unrealistic, the ideal scenario provides straightforward bounds on the detection performance achievable by the realistic scenario and enables an analytical expression for the performance gain provided by time reversal.

2) Time Reversal and Conventional Detection: We develop two approaches to the target in clutter detection problem: the conventional approach and the time reversal approach. In the conventional approach, the measurements are simply the direct measurements $Y\left(\omega_{q}\right)$. In the time reversal detection, besides the direct measurements $Y\left(\omega_{q}\right)$, we also have the time reversed measurements $X\left(\omega_{q}\right)$. We study conventional detection so that we can benchmark the detection gain, if any, provided by time reversal detection. In terms of the measurement protocol, it reduces to the probing step 1 . As with time reversal, we will consider two scenarios: 1) ideal, where we know the target channel response $\mathbf{h}_{t}$; and 2) realistic, where we do not know the target channel response $\mathbf{h}_{t}$.

3) Detectors: We have then four detection problems. The next four sections consider the following detectors:

1) conventional detector channel matched filter (CDCMF) for the ideal conventional detection problem;

2) time reversal channel matched filter (TRCMF) for the ideal time reversal detection problem;

3) change detection generalized likelihood ratio test (CD-GLRT) or energy detector (ED) for the realistic conventional detection problem;

4) time reversal generalized likelihood ratio test (TR-GLRT) for the realistic time reversal detection problem.

The first two detectors, CDCMF and TRCMF, and the last two detectors, ED (also called CD-GLRT) and TR-GLRT, are the optimal detectors and the generalized likelihood ratio detectors for the corresponding detection problems. Next, we will state each of these detection problems formally, then determine the corresponding likelihood ratio test statistic, the probability of false alarm $P_{\mathrm{FA}}$, the threshold $\eta$, and, the probability of detection $P_{\mathrm{D}}$. Before we do this, we recall a few preliminaries needed. a) Preliminaries: The likelihood ratio test statistic $\ell$ is [21],

$$
\ell(\mathbf{z})=\frac{p\left(\mathbf{z} \mid \mathbb{H}_{1}\right)}{p\left(\mathbf{z} \mid \mathbb{H}_{0}\right)}=\frac{\prod_{m=1}^{M} p\left(\mathbf{z}_{m} \mid \mathbb{H}_{1}\right)}{\prod_{m=1}^{M} p\left(\mathbf{z}_{m} \mid \mathfrak{H}_{0}\right)}
$$

where $p\left(\mathbf{z} \mid \mathbb{H}_{0}\right)$ and $p\left(\mathbf{z} \mid \mathbb{H}_{1}\right)$ are the probability density functions of the data conditioned on $\mathbb{H}_{0}$ and $\mathbb{H}_{1}$, respectively. The factorization on the right-hand side of (37) follows because conditioned on either hypothesis the measurements for different snapshots are independent.

Recall the definitions of $P_{\mathrm{FA}}$ and $P_{\mathrm{D}}$. If $\eta$ is the threshold

$$
\begin{aligned}
P_{\mathrm{FA}} & =\int_{\eta}^{\infty} p_{\ell}\left(L \mid \mathbb{H}_{0}\right) d L \\
P_{\mathrm{D}} & =\int_{\eta}^{\infty} p_{\ell}\left(L \mid \mathbb{H}_{1}\right) d L
\end{aligned}
$$

where $p_{\ell}\left(L \mid \mathbb{H}_{0}\right)$ and $p_{\ell}\left(L \mid \mathbb{H}_{1}\right)$ are the probability density functions of the test statistic $\ell$ under the null hypothesis $\mathbb{H}_{0}$ and the alternative hypothesis $\mathbb{H}_{1}$, respectively. We use the error function

$$
\operatorname{erf}(x)=\frac{2}{\sqrt{\pi}} \int_{0}^{x} e^{-t^{2}} d t, \quad x \in \mathcal{R}
$$

\section{B. Ideal Conventional Detection: Channel Matched Filter (CDCMF)}

We start by studying the conventional approach to the target in clutter detection problem. We use the equivalent formulation presented in Section III-A.

1) Detection Problem: The ideal conventional detection problem is equivalent to the following binary hypothesis problem:

$$
\begin{aligned}
& \mathfrak{H}_{1}: \mathbf{y}=\mathbf{S h}_{t}+\mathbf{v} \\
& \mathbb{H}_{0}: \mathbf{y}=\mathbf{v} .
\end{aligned}
$$

We recall that, in this ideal scenario, $\mathbf{h}_{t}$ in (41) is known.

The data probability density functions (pdfs) $p\left(\mathbf{y} \mid \mathbb{H}_{1}\right)$ and $p\left(\mathbf{y} \mid \mathbb{H}_{0}\right)$ conditioned on $\mathbb{H}_{1}$ and $\mathbb{H}_{0}$ follow from (33) and (29) and are, respectively [see, e.g., (1) for the expression of complex Gauss pdfs]

$$
\begin{aligned}
& p\left(\mathbf{y} \mid \mathbb{H}_{1}\right)=\prod_{m=1}^{M} \frac{1}{\pi^{Q}\left(\sigma_{v}^{2}\right)^{Q}} e^{-\frac{\left\|\mathbf{y} m-\mathbf{S h}_{t}\right\|^{2}}{\sigma_{v}^{2}}} \\
& p\left(\mathbf{y} \mid \mathbb{H}_{0}\right)=\prod_{m=1}^{M} \frac{1}{\pi^{Q}\left(\sigma_{v}^{2}\right)^{Q}} e^{-\frac{\left\|\mathbf{y} m_{m}\right\|^{2}}{\sigma_{v}^{2}}}
\end{aligned}
$$

where we used the conditional independence assumption of the data snapshots.

2) Likelihood Ratio Test $\ell_{\mathrm{CDCMF}}(\mathbf{x})$ : Replacing (42) and (43) in (37), taking the logarithm of the resulting expression, discarding the constant terms, and normalizing by the constant $2 \sigma_{v}\left\|\mathbf{S h}_{t}\right\|$ yields the linear statistic for the CDCMF detector

$$
\ell_{\mathrm{CDCMF}}(\mathbf{y})=\mathfrak{R}\left(\frac{\left(\mathbf{S h}_{t}\right)^{H} \sum_{m=1}^{M} \mathbf{y}_{m}}{\sigma_{v}\left\|\mathbf{S} \mathbf{h}_{t}\right\|}\right) .
$$


The test statistic (44) shows that the detector uses the knowledge of the target channel response-the detector is a (target) channel matched filter, i.e., it is matched to the known signal component $\mathbf{S h}_{t}$ at the output of the channel.

3) Probability of False Alarm $P_{\mathrm{FA}, \mathrm{CDCMF}}$ : In the null hypothesis $\mathbb{H}_{0}$, standard manipulations show that the variable inside the parenthesis in (44) is a circular Gaussian complex variable $\mathcal{C N}(0, M)$. This leads to

$$
\ell_{\mathrm{CDCMF}}(\mathbf{y}) \sim \mathcal{N}(0, M / 2)
$$

From (38) and (45), the probability of false alarm $P_{\mathrm{FA}, \mathrm{CDCMF}}$ for the CDCMF-detector is

$$
\begin{aligned}
P_{\mathrm{FA}, \mathrm{CDCMF}} & =\int_{\eta_{\mathrm{CDCMF}}}^{\infty} \frac{1}{\sqrt{2 \pi M / 2}} e^{-\frac{t^{2}}{2 M / 2}} d t \\
& =\int_{\frac{\eta_{\mathrm{CDCMF}}}{\sqrt{M}}}^{\infty} \frac{1}{\sqrt{\pi}} e^{-t^{2}} d t .
\end{aligned}
$$

Using the error function in (40), $P_{\mathrm{FA}, \mathrm{CDCMF}}$ is compactly written as

$$
P_{\mathrm{FA}, \mathrm{CDCMF}}=\frac{1}{2}\left(1-\operatorname{erf}\left(\frac{\eta_{\mathrm{CDCMF}}}{\sqrt{M}}\right)\right) .
$$

Threshold $\eta_{\mathrm{CDCMF}}$ : From (47), the detection threshold is

$$
\eta_{\mathrm{CDCMF}}=\sqrt{M} \operatorname{erf}^{-1}\left(1-2 P_{\mathrm{FA}, \mathrm{CDCMF}}\right)
$$

where $\operatorname{erf}^{-1}(\cdot)$ is the inverse error function.

4) Probability of Detection $P_{\mathrm{D}, \mathrm{CDCMF}}$ : In the alternative hypothesis $\mathbb{H}_{1}$, standard manipulations show that the quantity in parenthesis in the expression of the decision statistic (44) is $\mathcal{C N}\left(d_{1}, M\right)$, where

$$
d_{1}=M \frac{\left\|\mathbf{S h}_{t}\right\|}{\sigma_{v}}=M \frac{\sqrt{\sum_{q=0}^{Q-1}\left|S\left(\omega_{q}\right) H_{t}\left(\omega_{q}\right)\right|^{2}}}{\sigma_{v}} .
$$

The pdf of the test statistic under $\mathbb{H}_{1}$ is then

$$
\ell_{\mathrm{CDCMF}}(\mathbf{y}) \sim \mathcal{N}\left(d_{1}, M / 2\right)
$$

The detection probability $P_{\mathrm{D}, \mathrm{CDCMF}}$ follows from (39) and, by making use of the error function (40), it is simply

$$
\begin{aligned}
& P_{\mathrm{D}, \mathrm{CDCMF}}=\frac{1}{2}\left(1-\operatorname{erf}\left(\frac{\eta_{\mathrm{CDCMF}}-d_{1}}{\sqrt{M}}\right)\right) \\
& =\frac{1}{2}-\frac{1}{2} \operatorname{erf}\left(\operatorname{erf}^{-1}\left(1-2 P_{\mathrm{FA}, \mathrm{CDCMF}}\right)-\frac{d_{1}}{\sqrt{M}}\right) .
\end{aligned}
$$

\section{Ideal Time Reversal: Channel Matched Filter (TRCMF)}

1) Detection Problem: Because the target channel response $\mathbf{h}_{t}$ is assumed known, we need to consider only the data $X_{m}\left(\omega_{q}\right)$ and $\mathbf{x}$ received under the time reversal step 1 . These signals are modified from (13) and (35) by assuming the noise $\mathbf{v}=\mathbf{0}$ under the alternative hypothesis and by setting all energy normalization factors to the deterministic known constant

$$
k_{m} \equiv k=\sqrt{\frac{Q E_{s}}{\sum_{q=0}^{Q-1}\left\|S\left(\omega_{q}\right)\right\|^{2}\left\|H_{t}\left(\omega_{q}\right)\right\|^{2}}} .
$$

The detection problem is equivalent to

$$
\begin{aligned}
& \mathfrak{H}_{1}: \mathbf{x}=k \mathbf{S}^{*} \mathbf{h}_{t}^{*} \odot \mathbf{h}_{t}+\mathbf{w} \\
& \mathfrak{H}_{0}: \mathbf{x}=\mathbf{w} .
\end{aligned}
$$

Note that, because $\mathbf{h}_{t}$ is assumed known, the transmitted signal $k \mathbf{S}^{*} \mathbf{h}_{t}^{*}$ can be generated by the transmitter with no need for the probing step 1 . The data pdfs under $\mathbb{H}_{1}$ and $\mathbb{H}_{0}$ follow from the assumptions on $\mathbf{w}_{m}$

$$
\begin{aligned}
& p\left(\mathbf{x} \mid \mathfrak{H}_{1}\right)=\prod_{m=1}^{M} \frac{1}{\pi^{Q}\left(\sigma_{w}^{2}\right)^{Q}} e^{-\frac{\left\|\mathbf{x}_{m}-k \mathbf{y}_{t}^{*} \odot \mathbf{h}_{t}\right\|^{2}}{\sigma_{w}^{2}}} \\
& p\left(\mathbf{x} \mid \mathfrak{H}_{0}\right)=\prod_{m=1}^{M} \frac{1}{\pi^{Q}\left(\sigma_{w}^{2}\right)^{Q}} e^{-\frac{\left\|\mathbf{x}_{m}\right\|^{2}}{\sigma_{w}^{2}}} .
\end{aligned}
$$

2) Likelihood Ratio Test $\ell_{\mathrm{TRCMF}}(\mathbf{x})$ : Replacing these expressions in the expression of the likelihood ratio (37), taking the logarithm, discarding constant known terms, and normalizing the test statistic by the known quantity $2 \sigma_{w}\left\|k \mathbf{y}_{t}^{*} \odot \mathbf{h}_{t}\right\|$, yields the linear test statistic

$$
\ell_{\mathrm{TRCMF}}(\mathbf{x})=\mathfrak{R}\left(\frac{\left(\mathbf{y}_{t}^{*} \odot \mathbf{h}_{t}\right)^{H} \sum_{m=1}^{M} \mathbf{x}_{m}}{\sigma_{w}\left\|\mathbf{y}_{t}^{*} \odot \mathbf{h}_{t}\right\|}\right) .
$$

3) Probability of False Alarm $P_{\mathrm{PA}, \mathrm{TRCMF}}$ : The test statistic given by (56) is linear and, given the assumptions on the noise $\mathbf{w}_{m}$, it can be shown that the quantity inside $\mathfrak{R}(\cdot)$ in (56) is a complex random variable with probability density function $\mathcal{C N}(0, M)$. This implies [20] that

$$
\ell_{\mathrm{TRCMF}}(\mathbf{x}) \sim \mathcal{N}(0, M / 2) .
$$

Just like for the CDCMD detector, we find that $P_{\mathrm{FA}, \mathrm{TRCMF}}$ is

$$
P_{\mathrm{FA}, \mathrm{TRCMF}}=\frac{1}{2}\left(1-\operatorname{erf}\left(\frac{\eta_{\mathrm{TRCMF}}}{\sqrt{M}}\right)\right)
$$

which is exactly like (47).

4) Threshold $\eta_{\mathrm{TRCMF}}$ : The threshold $\eta_{\mathrm{TRCMF}}$ for the TRCMF detector follows by inverting (48)

$$
\eta_{\mathrm{TRCMF}}=\sqrt{M} \operatorname{erf}^{-1}\left(1-2 P_{\mathrm{FA}, \mathrm{TRCMF}}\right) .
$$

5) Probability of Detection $P_{\mathrm{D}, \mathrm{TRCMF}}$ : It is straightforward to show that, conditioned on $\mathfrak{H}_{1}$, the pdf of $\ell_{\mathrm{TRCMF}}(\mathbf{x})$ is

$$
\ell_{\mathrm{TRCMF}}(\mathbf{x}) \sim \mathcal{N}\left(d_{2}, M / 2\right)
$$

where

$$
\begin{aligned}
d_{2} & =M k \frac{\left\|\mathbf{y}_{t}^{*} \odot \mathbf{h}_{t}\right\|}{\sigma_{w}} \\
& =M k \frac{\sqrt{\sum_{q=0}^{Q-1}\left|S\left(\omega_{q}\right)\right|^{2}\left|H_{t}\left(\omega_{q}\right)\right|^{4}}}{\sigma_{w}} .
\end{aligned}
$$


The detection probability is obtained as for the CDCMD detector. We get

$$
\begin{aligned}
& P_{\mathrm{D}, \mathrm{TRCMF}} \\
& \quad=\frac{1}{2}\left(1-\operatorname{erf}\left(\frac{\eta-d_{2}}{\sqrt{M}}\right)\right) \\
& \quad=\frac{1}{2}-\frac{1}{2} \operatorname{erf}\left(\operatorname{erf}^{-1}\left(1-2 P_{\mathrm{FA}, \mathrm{TRCMF}}\right)-\frac{d_{2}}{\sqrt{M}}\right) .
\end{aligned}
$$

\section{Realistic Conventional Detection: Energy Detector}

1) Detection Problem: We now consider the conventional detection problem when we do not know the target channel response $\mathbf{h}_{t}$. The setup of the problem is like in (41) for ideal conventional detection, except that now $\mathbf{h}_{t}$ is unknown. The data pdfs $p\left(\mathbf{y} \mid \mathbb{H}_{1}\right)$ and $p\left(\mathbf{y} \mid \mathbb{H}_{0}\right)$ under $\mathbb{H}_{1}$ and $\mathbb{H}_{0}$ are given again as in (42) and (43), respectively.

2) Likelihood Ratio Test $\ell_{\mathrm{ED}}(\mathbf{y})$ : Because $\mathbf{h}_{t}$ is unknown, we adopt as detector the generalized likelihood ratio test (GLRT)

$$
\frac{\max _{\mathbf{h}_{t}} p\left(\mathbf{y} \mid \mathbb{H}_{1}\right)}{p\left(\mathbf{y} \mid \mathbb{H}_{0}\right)}
$$

We could refer to this detector as the change detection generalized likelihood ratio detector (CD-GLRT). However, as will be shown below, the detector has an energy detector-like structure. Thus, we refer to this detector as the energy detector (ED).

The maximum in the numerator of (63) is at the maximum likelihood estimate of $\mathbf{h}_{t}$ under $\mathbb{H}_{1}$

$$
\hat{\mathbf{h}}_{t}=\arg \max _{\mathbf{h}_{t}} p\left(\mathbf{y} \mid \mathbb{H}_{1}\right) .
$$

This yields

$$
\hat{\mathbf{h}}_{t}=\mathbf{S}^{-1} \frac{1}{M} \sum_{1}^{M} \mathbf{y}_{m}
$$

Using (64) in (63), taking the logarithm, neglecting constants, yields

$$
\begin{aligned}
\sum_{m=1}^{M} & \left(\left\|\mathbf{y}_{m}\right\|^{2}-\left\|\mathbf{y}_{m}-\mathbf{S} \hat{\mathbf{h}}_{t}\right\|^{2}\right) \\
& =\sum_{m=1}^{M}\left(2 \mathfrak{R}\left\{\left(\mathbf{S} \hat{\mathbf{h}}_{t}\right)^{H} \mathbf{y}_{m}\right\}-\left\|\mathbf{S} \hat{\mathbf{h}}_{t}\right\|^{2}\right) \\
& =2 \mathfrak{R}\left\{\left(\mathbf{S} \hat{\mathbf{h}}_{t}\right)^{H} \sum_{m=1}^{M} \mathbf{y}_{m}\right\}-M\left\|\mathbf{S} \hat{\mathbf{h}}_{t}\right\|^{2} \\
& =M\left\|\mathbf{S} \hat{\mathbf{h}}_{t}\right\|^{2} \\
& =\frac{\left\|\sum_{m=1}^{M} \mathbf{y}_{m}\right\|^{2}}{M} .
\end{aligned}
$$

Normalizing (65) by $\sigma_{v}^{2} / 2$ results finally in

$$
\ell_{\mathrm{ED}}(\mathbf{y})=\frac{1}{M \sigma_{v}^{2} / 2}\left\|\sum_{m=1}^{M} \mathbf{y}_{m}\right\|^{2} .
$$

3) Probability of False Alarm $P_{\mathrm{FA}, \mathrm{ED}}(\mathbf{y})$ : In the null hypothesis $\mathbb{H}_{0}$ and noise only case, the test statistic for the energy detector is given by

$$
\ell_{\mathrm{ED}}(\mathbf{y})=\sum_{q=0}^{Q-1} \frac{\left|\sum_{m=1}^{M} V_{m}\left(\omega_{q}\right)\right|^{2}}{M \sigma_{v}^{2} / 2}
$$

where, like before, $V_{m}\left(\omega_{q}\right) \sim \mathcal{C N}\left(0, \sigma_{v}^{2}\right), q=0, \ldots, Q-$ $1, m=1, \ldots M$ are circular complex Gaussian random variables. Since the real and imaginary parts of $V_{m}\left(\omega_{q}\right)$ are independent and each of them is $\mathcal{N}\left(0, \sigma_{v}^{2} / 2\right)$, this implies that each term in the sum in (67)

$$
\frac{\sum_{m=1}^{M} V_{m}\left(\omega_{q}\right)}{\sqrt{M \sigma_{v}^{2} / 2}} \sim \mathcal{C N}(0,2)
$$

Therefore $\left|\sum_{m=1}^{M} V_{m}\left(\omega_{q}\right)\right|^{2} /\left(M \sigma_{v}^{2} / 2\right)$ is the sum of the squares of two $\mathcal{N}(0,1)$ random variables, and so a central $\chi$-square distribution with two degrees of freedom. This implies that $\ell_{\mathrm{ED}}(\mathbf{y})$ has a central $\chi$-square distribution with $2 Q$ degrees of freedom

$$
\ell_{\mathrm{ED}}(\mathbf{y}) \sim \chi_{2 Q}^{2}(0) .
$$

From (68), we compute the probability of false alarm $P_{\mathrm{FA}, \mathrm{ED}}$. Let $\psi_{\nu, \mu}(x)$ denote the cumulative distribution function of a noncentral $\chi$-square random variable with $\nu$ degrees of freedom and noncentrality parameter $\mu$. Then $P_{\mathrm{FA}, \mathrm{ED}}$ is

$$
P_{\mathrm{FA}, \mathrm{ED}}=1-\psi_{2 Q, 0}\left(\eta_{\mathrm{ED}}\right)
$$

where $\eta_{\mathrm{ED}}$ is the threshold.

4) Threshold $\eta_{\mathrm{ED}}$ : Inverting (69) gives the threshold $\eta_{\mathrm{ED}}$ for the energy detector as

$$
\eta_{\mathrm{ED}}=\psi_{2 Q, 0}^{-1}\left(1-P_{\mathrm{FA}, \mathrm{ED}}\right)
$$

where $\psi_{\nu, \mu}^{-1}(\cdot)$ is the inverse function of the cumulative distribution $\psi_{\nu, \mu}(\cdot)$.

5) Probability of Detection $P_{\mathrm{D}, \mathrm{ED}}$ : The test statistic under the alternative hypothesis $\mathbb{H}_{1}$ is

$$
\ell_{\mathrm{ED}}(\mathbf{y})=\sum_{q=0}^{Q-1} \frac{\left|\sum_{m=1}^{M} Y_{m}\left(\omega_{q}\right)\right|^{2}}{M \sigma_{v}^{2} / 2}
$$

where $Y_{m}\left(\omega_{q}\right) \sim \mathcal{C N}\left(S\left(\omega_{q}\right) H_{t}\left(\omega_{q}\right), \sigma_{v}^{2}\right), q=0, \ldots, Q-$ $1, m=1, \ldots, M$. Each term

$$
\frac{\left|\sum_{m=1}^{M} Y_{m}\left(\omega_{q}\right)\right|^{2}}{M \sigma_{v}^{2} / 2} \sim \chi_{2}^{2}\left(\frac{2 M\left|S\left(\omega_{q}\right)\right|^{2}\left|H_{t}\left(\omega_{q}\right)\right|^{2}}{\sigma_{v}^{2}}\right)
$$


i.e., it is noncentral $\chi$-square distributed with two degrees of freedom. It follows that $\ell_{\mathrm{ED}}(\mathbf{y})$ is noncentral $\chi$-square distributed with $2 Q$ degrees of freedom

$$
\ell_{\mathrm{ED}}(\mathbf{y}) \sim \chi_{2 Q}^{2}(\mu)
$$

where the noncentral parameter is given by

$$
\mu=2 M \sum_{q=0}^{Q-1}\left|S\left(\omega_{q}\right)\right|^{2}\left|H_{t}\left(\omega_{q}\right)\right|^{2} / \sigma_{v}^{2} .
$$

Hence, the detection probability $P_{\mathrm{D}, \mathrm{ED}}$ for the energy detector takes the form

$$
\begin{aligned}
P_{\mathrm{D}, \mathrm{ED}} & =1-\psi_{(2 Q, \mu)}\left(\eta_{\mathrm{ED}}\right) \\
& =1-\psi_{(2 Q, \mu)}\left(\psi_{(2 Q, 0)}^{-1}\left(1-P_{\mathrm{FA}, \mathrm{ED}}\right)\right) .
\end{aligned}
$$

The probabilities of false alarm $P_{\mathrm{FA}, \mathrm{ED}}$ and detection $P_{\mathrm{D}, \mathrm{ED}}$, and the threshold $\eta_{\mathrm{ED}}$ can be found by standard approximations to the $\chi$-square distribution as found, for example, in [23] or as tabulated in standard scientific computation packages.

\section{E. Realistic Time Reversal: Generalized Likelihood Ratio Test (TR-GLRT)}

1) Detection Problem: The detection problem is now the following:

$$
\begin{aligned}
& \mathbb{H}_{1}: \mathbf{z}=\left[\begin{array}{c}
\mathbf{y}_{t}^{*} \\
\mathbf{x}_{t}
\end{array}\right]+\left[\begin{array}{l}
\mathbf{v}^{*} \\
\mathbf{w}
\end{array}\right] \\
& \mathbb{H}_{0}: \mathbf{z}=\left[\begin{array}{l}
\mathbf{v}^{*} \\
\mathbf{w}
\end{array}\right]
\end{aligned}
$$

where $\mathbf{y}_{t}$ and $\mathbf{x}_{t}$ are the means given in (33) and in (35). It is important to note that in the detection formulation in (76), we have conjugated the data received in the probing step $\mathbf{y}_{t}$. This is of course an information-preserving transformation, so no loss or gain of information is achieved. However, it greatly simplifies the target channel response estimate as we will see below. The detection problem in (76) is difficult to study analytically. We consider the approximate problem where we neglect the information provided by the energy normalization factors $k_{m}$. In the study of this detector, we will take $k_{m}$ to be deterministic. This is actually a good approximation. In our simulations in Section $\mathrm{V}$, we will observe that $k_{m}$ has small variability. Also, we have performed a noise analysis elsewhere that shows that the second order moment of $k_{m}$ is small in either the high or low signal-to-noise ratio (SNR) regimes.

Let

$$
\Omega(q)=\left[\begin{array}{cc}
\sigma_{v}^{2} & k_{m} H_{t}^{*}\left(\omega_{q}\right) \sigma_{v}^{2} \\
k_{m} H_{t}\left(\omega_{q}\right) \sigma_{v}^{2} & k_{m}^{2}\left|H_{t}\left(\omega_{q}\right)\right|^{2} \sigma_{v}^{2}+\sigma_{w}^{2}
\end{array}\right] .
$$

The pdfs $p\left(\mathbf{z} ; \mathbf{h}_{t} \mid \mathbb{H}_{1}\right)$ and $p\left(\mathbf{z}_{m} \mid \mathbb{H}_{0}\right)$ conditioned on $k_{m}$ under hypotheses $\mathbb{H}_{1}$ and $\mathbb{H}_{0}$ are given by

$$
\begin{aligned}
p\left(\mathbf{z} ; \mathbf{h}_{t} \mid \mathbb{H}_{1}\right) & \\
= & \prod_{m=1}^{M} \prod_{q=0}^{Q-1} \frac{1}{\pi|\Omega(q)|} \\
& \times \exp \left\{-\left[\begin{array}{c}
Y_{m}^{*}\left(\omega_{q}\right)-S^{*}\left(\omega_{q}\right) H_{t}^{*}\left(\omega_{q}\right) \\
X_{m}\left(\omega_{q}\right)-k_{m}\left|H_{t}\left(\omega_{q}\right)\right|^{2} S^{*}\left(\omega_{q}\right)
\end{array}\right]^{H} \Omega^{-1}(q)\right. \\
& {\left.\left[\begin{array}{c}
Y_{m}^{*}\left(\omega_{q}\right)-S^{*}\left(\omega_{q}\right) H_{t}^{*}\left(\omega_{q}\right) \\
X_{m}\left(\omega_{q}\right)-k_{m}\left|H_{t}\left(\omega_{q}\right)\right|^{2} S^{*}\left(\omega_{q}\right)
\end{array}\right]\right\} } \\
= & \prod_{m=1}^{M} \prod_{q=0}^{Q-1} \frac{1}{\pi \sigma_{w}^{2} \sigma_{v}^{2}} \exp \left\{-\frac{1}{\sigma_{w}^{2} \sigma_{v}^{2}}\left(\left|Y_{m}\left(\omega_{q}\right)\right|^{2} k_{m}^{2} \sigma_{v}^{2}\right.\right. \\
& \left.+\left|S\left(\omega_{q}\right)\right|^{2} \sigma_{w}^{2}\right)\left|H_{t}\left(\omega_{q}\right)\right|^{2} \\
& +\frac{2}{\sigma_{w}^{2} \sigma_{v}^{2}} \mathfrak{R}\left\{\left[k_{m} X_{m}^{*}\left(\omega_{q}\right) Y_{m}^{*}\left(\omega_{q}\right) \sigma_{v}^{2}\right.\right. \\
& \left.\left.+\sigma_{w}^{2} Y_{m}^{*}\left(\omega_{q}\right) S\left(\omega_{q}\right)\right] H_{t}\left(\omega_{q}\right)\right\} \\
& \left.-\frac{1}{\sigma_{w}^{2} \sigma_{v}^{2}}\left(\left|X_{m}\left(\omega_{q}\right)\right|^{2} \sigma_{v}^{2}+\left|Y_{m}\left(\omega_{q}\right)\right|^{2} \sigma_{w}^{2}\right)\right\} \\
p\left(\mathbf{z} \mid \mathbb{H}_{0}\right) & \prod_{m=1}^{M} \prod_{q=0}^{Q-1} \frac{1}{\pi \sigma_{w}^{2} \sigma_{v}^{2}} \\
= & \times \exp \left\{-\frac{\left|X_{m}\left(\omega_{q}\right)\right|^{2}}{\sigma_{w}^{2}}\right\} \exp \left\{-\frac{\left|Y_{m}\left(\omega_{q}\right)\right|^{2}}{\sigma_{v}^{2}}\right\}
\end{aligned}
$$

2) Likelihood Ratio Test $\ell_{\mathrm{TR}-\mathrm{GLRT}}(\mathbf{z})$ : Like for the realistic conventional detection problem in Section III-D that led to the energy detector, here we do not know $\mathbf{h}_{t}$. We adopt again the generalized likelihood ratio test; see (63). Taking the logarithm of the ratio of the two pdfs (79) and (80) evaluated at the maximum likelihood estimate of $\mathbf{h}_{t}$, the test statistic is

$$
\begin{aligned}
\ell_{\mathrm{TR}-\mathrm{GLRT}}(\mathbf{z}) & \ln p\left(\mathbf{z} \mid \mathbb{H}_{1}\right)-\ln p\left(\mathbf{z} \mid \mathbb{H}_{0}\right) \\
= & \sum_{m=1}^{M} \sum_{q=0}^{Q-1}\left\{\frac{\left|X_{m}\left(\omega_{q}\right)\right|^{2}}{\sigma_{w}^{2}}+\frac{\left|Y_{m}\left(\omega_{q}\right)\right|^{2}}{\sigma_{v}^{2}}\right. \\
& -\frac{1}{\sigma_{v}^{2} \sigma_{w}^{2}}\left(\left|Y_{m}\left(\omega_{q}\right)\right|^{2} k_{m}^{2} \sigma_{v}^{2}+\left|S\left(\omega_{q}\right)\right|^{2} \sigma_{w}^{2}\right)\left|\widehat{H}_{t}\left(\omega_{q}\right)\right|^{2} \\
& +\frac{2}{\sigma_{v}^{2} \sigma_{w}^{2}} \mathfrak{R}\left\{\left[k_{m} X_{m}^{*}\left(\omega_{q}\right) Y_{m}^{*}\left(\omega_{q}\right) \sigma_{v}^{2}\right.\right. \\
& \left.\left.+\sigma_{w}^{2} Y_{m}^{*}\left(\omega_{q}\right) S\left(\omega_{q}\right)\right] \widehat{H}_{t}\left(\omega_{q}\right)\right\} \\
& \left.-\frac{1}{\sigma_{v}^{2} \sigma_{w}^{2}}\left(\left|X_{m}\left(\omega_{q}\right)\right|^{2} \sigma_{v}^{2}+\left|Y_{m}\left(\omega_{q}\right)\right|^{2} \sigma_{w}^{2}\right)\right\} \\
= & -\frac{1}{\sigma_{v}^{2} \sigma_{w}^{2}} \sum_{m=1}^{M-1} \sum_{q=0}^{Q-1}\left(\left|Y_{m}\left(\omega_{q}\right)\right|^{2} k_{m}^{2} \sigma_{v}^{2}\right. \\
& \left.+\left|S\left(\omega_{q}\right)\right|^{2} \sigma_{w}^{2}\right)\left|\widehat{H}_{t}\left(\omega_{q}\right)\right|^{2} \\
& -2 \mathfrak{R}\left\{\left[k_{m} X_{m}^{*}\left(\omega_{q}\right) Y_{m}^{*}\left(\omega_{q}\right) \sigma_{v}^{2}\right.\right. \\
& \left.\left.\left.+\sigma_{w}^{2} Y_{m}^{*}\left(\omega_{q}\right) S\left(\omega_{q}\right)\right] \widehat{H}_{t}\left(\omega_{q}\right)\right\}\right]
\end{aligned}
$$


where $\hat{H}_{t}\left(\omega_{q}\right)$ is the maximum likelihood estimate of $H_{t}\left(\omega_{q}\right)$ to be determined below. This is not a linear test statistic, which is to be expected given that the channel is no longer known.

3) Maximum Likelihood (ML) Estimate $\widehat{\mathbf{h}_{t}}$ Under $\mathbb{H}_{1}$ : We derive the maximum likelihood estimate of $\mathbf{h}_{t}$ under $\mathbb{H}_{1}$. Like before, we neglect the dependency of the energy normalization factors $k_{m}$ on the target channel response and so it is only an approximation to the true ML estimate. Taking the partial derivative of $-\ln p\left(\mathbf{z} ; \mathbf{h}_{t} \mid \mathbb{H}_{1}\right)$ with respect to $H_{t}^{*}\left(\omega_{q}\right)$, and ignoring the constant terms yields, after noticing that $\left(\partial\left|H_{t}\left(\omega_{q}\right)\right|^{2} / \partial H_{t}^{*}\left(\omega_{q}\right)\right)=H_{t}\left(\omega_{q}\right),[24]$

$$
\begin{aligned}
& \frac{\partial\left[-\ln p\left(\mathbf{z} ; \mathbf{h}_{t} \mid \mathbb{H}_{1}\right)\right]}{\partial H_{t}^{*}\left(\omega_{q}\right)} \\
& =\frac{1}{\sigma_{v}^{2} \sigma_{w}^{2}} \sum_{m=1}^{M}\left[\left(\left|Y_{m}\left(\omega_{q}\right)\right|^{2} k_{m}^{2} \sigma_{v}^{2}+\left|S\left(\omega_{q}\right)\right|^{2} \sigma_{w}^{2}\right) H_{t}\left(\omega_{q}\right)\right. \\
& \left.\quad-\left(k_{m} X_{m}\left(\omega_{q}\right) Y_{m}\left(\omega_{q}\right) \sigma_{v}^{2}+Y_{m}\left(\omega_{q}\right) S^{*}\left(\omega_{q}\right) \sigma_{w}^{2}\right)\right]=0 .
\end{aligned}
$$

After dividing the numerator and the denominator by $\sigma_{v}^{2} \sigma_{w}^{2}$, we obtain

$$
\widehat{H}_{t}\left(\omega_{q}\right)=\frac{\sum_{m=1}^{M}\left[\frac{Y_{m}\left(\omega_{q}\right) S^{*}\left(\omega_{q}\right)}{\sigma_{v}^{2}}+\frac{k_{m} Y_{m}\left(\omega_{q}\right) X_{m}\left(\omega_{q}\right)}{\sigma_{w}^{2}}\right]}{\sum_{m=1}^{M}\left[\frac{\left|S\left(\omega_{q}\right)\right|^{2}}{\sigma_{v}^{2}}+\frac{\left|Y_{m}\left(\omega_{q}\right)\right|^{2} k_{m}^{2}}{\sigma_{w}^{2}}\right]} .
$$

Equation (84) completes the structure of the TR-GLRT test statistic. It is a surprisingly intuitively pleasing expression for the estimate of the target channel response. The fractions in the denominator are approximate channel input SNRs for the probing and time reversal steps, respectively, while the fractions in the numerator are approximately these SNRs normalized by the target channel response. If the noises $\mathbf{v}$ and $\mathbf{w}$ are small, the numerator is then approximately the denominator times $H_{t}\left(\omega_{q}\right)$, so that the right-hand side, and so the ML channel estimate, is close to the true value of the target channel response. A final note regarding the ML estimate (84) is that this intuitive expression results because we formulated the time reversal detection problem using the time reversed signal received in the probing step 1 .

In Section $\mathrm{V}$, we study the probabilities of false alarm $P_{\mathrm{FA}, \mathrm{TR}-\mathrm{GLRT}}$ and detection $P_{\mathrm{D}, \mathrm{TR}-\mathrm{GLRT}}$, and the threshold $\eta_{\text {TR-GLRT }}$ by Monte Carlo simulation since it cannot be determined analytically.

\section{Time ReVERsal Detection Gain}

We now quantify the performance gain provided by time reversal detection over conventional detection, i.e., what is the gain in performance achieved by the TRCMF over the CDCMF for the known target channel. We notice that, for both detectors, the threshold under a fixed false alarm probability is exactly the same; see (48) and (59). This observation allows us to compare the two detectors by computing the ratio of $d_{2}^{2}$ and $d_{1}^{2}$ defined in
(61) and (49), respectively [21], [22]. In other words, the SNR gain (SNRG) provided by time reversal is

$$
\mathrm{SNRG}=\frac{d_{2}^{2}}{d_{1}^{2}}=\frac{\sum_{q=0}^{Q-1}\left|S\left(\omega_{q}\right)\right|^{2} \sum_{q=0}^{Q-1}\left|S\left(\omega_{q}\right)\right|^{2}\left|H_{t}\left(\omega_{q}\right)\right|^{4}}{\left(\sum_{q=0}^{Q-1}\left|S\left(\omega_{q}\right)\right|^{2}\left|H_{t}\left(\omega_{q}\right)\right|^{2}\right)^{2}}
$$

where the signal energy is $E_{s}=(1 / Q) \sum_{q=0}^{Q-1}\left|S\left(\omega_{q}\right)\right|^{2}$ and we assumed that $\sigma_{v}^{2}=\sigma_{w}^{2}$. We have the following result.

Result 1: The SNRG of the time reversal matched filter over the conventional matched filter is

$$
\mathrm{SNRG} \geq 1 .
$$

Equality holds when $\left\{\forall q:\left|H_{t}\left(\omega_{q}\right)\right|=a \in \mathcal{R}^{+}\right\}$, where $a$ is a nonnegative constant.

Proof: The result follows by direct application of Schwartz inequality. We can factor the denominator in (86) as

$$
\begin{aligned}
{[\sum_{q=0}^{Q-1} \underbrace{\left|S\left(\omega_{q}\right)\right|}_{\left|f_{q}\right|} \underbrace{\left|S\left(\omega_{q}\right)\right|\left|H_{t}\left(\omega_{q}\right)\right|^{2}}_{\left|g_{q}\right|}]^{2} } \\
\leq \sum_{q=0}^{Q-1} \underbrace{\left|S\left(\omega_{q}\right)\right|^{2}}_{\left|f_{q}\right|^{2}} \sum_{q=0}^{Q-1} \underbrace{\left|S\left(\omega_{q}\right)\right|^{2}\left|H_{t}\left(\omega_{q}\right)\right|^{4}}_{\left|g_{q}\right|^{2}}
\end{aligned}
$$

with equality when

$$
\left|S\left(\omega_{q}\right)\right|\left|H_{t}\left(\omega_{q}\right)\right|^{2}=a^{2}\left|S\left(\omega_{q}\right)\right| .
$$

There are a number of interesting observations we can make regarding Result 1.

1) Time Reversal Gain: Equation (86) shows that the TRCMF has a net performance gain over the CDCMF. How large this gain is depends on the target channel response $\mathbf{h}_{t}$. For instance, for a flat channel, e.g., single point scatterer and no multipath, where $\left|H_{t}\left(\omega_{q}\right)\right|=a, a \geq 0$ is a constant, $\mathrm{SNRG}=1$. When the target response has large variations across a frequency range as induced by a rich scattering environment, the gain can be very significant. This observation will be experimentally verified in Section V, where we measure the target channel response for real electromagnetic channels and compute SNRG.

2) Time Reversal: Joint Optimization at the Receiver and the Transmitter: Both detectors, the time reversal TRCMF and the conventional CDCMF, are perfectly matched to the (noiseless) signal at the output of the channel, i.e., they are channel matched. They are optimal for their corresponding detection problems. The performance gain of the time reversal matched filter detector over the conventional matched filter detector is the result of the implicit optimization achieved by time reversal at both the transmitter and the receiver. However, besides optimizing the SNR at the receiver, the TRCMF detector also optimizes automatically the signal at the transmitter. 
3) Time Reversal-Waveform Reshaping: The time reversal detection gain can be explained by the automatic reshaping of the signal achieved by the transmitter, which adjusts better the transmitted signal to the target channel frequency response. The target channel frequency response is induced by the scattering environment since the backscatter from the target is not simply the direct path from the target to the receiver but is also the secondary scattering from the scatterers to the target and then from the target to the receiver. A richer scattering environment induces a richer target response.

4) Time Reversal and Target Channel Type: There are potentially large gains to be achieved by time reversal. To see this, we rework the expression of the gain. We discuss the simpler case where the transmitted signal is a (time domain) sinc pulse, so $S\left(\omega_{q}\right) \equiv 1, \forall q$. Then the gain (86) can be rewritten as

$$
\begin{aligned}
\text { SNRG } & =\frac{Q \sum_{q=0}^{Q-1}\left|H_{t}\left(\omega_{q}\right)\right|^{4}}{\left(\sum_{q=0}^{Q-1}\left|H_{t}\left(\omega_{q}\right)\right|^{2}\right)^{2}} \\
& =\frac{1}{Q} \sum_{q=0}^{Q-1}\left|H_{t}\left(\omega_{q}\right)\right|^{4}\left(\frac{1}{Q} \sum_{q=0}^{Q-1}\left|H_{t}\left(\omega_{q}\right)\right|^{2}\right)^{2} .
\end{aligned}
$$

We define the target channel type 4 as the empirical distribution of the (magnitude) of the target channel response. If we consider the empirical distribution, i.e., the normalized histogram, of the values of the target channel response $\left|H_{t}\left(\omega_{q}\right)\right|,(89)$ is interpreted as the ratio of the fourth-order absolute moment $\mu_{4}$ over the square of the second-order absolute moment $\mu_{2}^{2}$ of the empirical distribution or target channel type

$$
\gamma=\frac{\mu_{4}}{\mu_{2}^{2}}
$$

The ratio $\gamma$ is not the kurtosis $\kappa$, which is the ratio of the fourthorder centered moment over the square of the variance. We will compute $\gamma$ for real channels in Section V. Here, we get an intuitive feeling for SNRG by looking at the value of the kurtosis $\kappa$ for a few distributions for which it is readily available. For a normal random variable, $\kappa=3=4.7 \mathrm{~dB}$. Of interest will be leptokurtic ${ }^{5}$ distributions. For example, the Laplace (or double-sided exponential) standard distribution has $\kappa=6=$ $7.78 \mathrm{~dB}$, while the student or $t$-distribution with five degrees of freedom has $\kappa=9=9.54 \mathrm{~dB}$.

\section{Performance Study: ExPerimental Results}

This section studies with a mix of real electromagnetic (EM) data and simulated noise the performance gain provided by time reversal detection over conventional detection. We recall from Section III that the CDCMF and ED address the conventional detection problem (41) where no time reversal occurs, while the TRCMF and TR-GLRT consider the time reversal detection

\footnotetext{
${ }^{4}$ As noted in Section I, the expression type is used as an information theoretic concept to refer to the empirical distribution [see [1]] of the channel frequency response.

${ }^{5}$ Leptokurtic distributions have a positive kurtosis excess, i.e., a kurtosis larger than three, the kurtosis of the normal distribution.
}

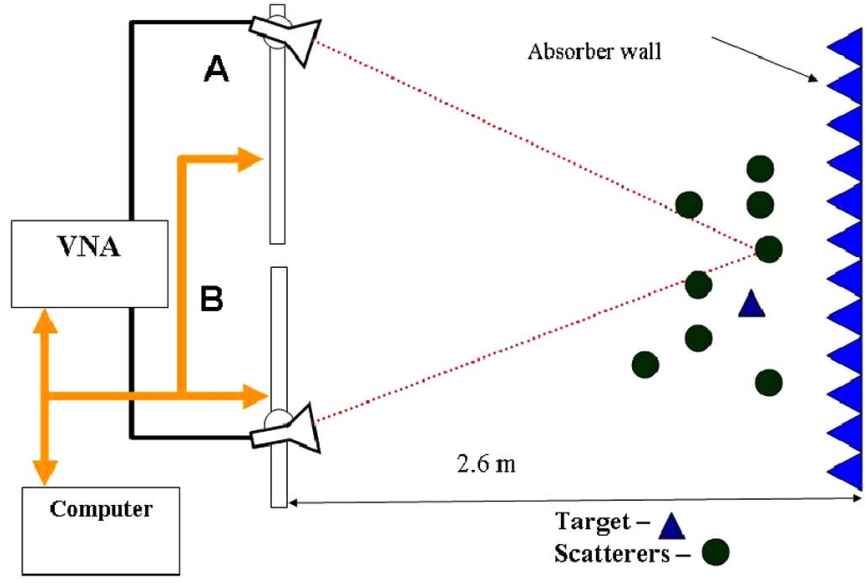

Fig. 1. Channel I: Free space. Transmit antenna A and receive antenna B are horn antennas. Operating frequency range is $4-6 \mathrm{GHz}$. Scatterers are a mixture of 20 copper and solid dielectric pipes, represented as circles. The target is a copper pipe, represented as a triangle.

problem (76) where the time reversed backscattered signals are retransmitted. Also, the CDCMF and the TRCMF, which are channel matched, assume full knowledge of the target channel response $H_{t}\left(\omega_{q}\right)$, while the ED and TR-GLRT have no knowledge of the channel and are the generalized likelihood ratio tests for the corresponding problems. Accordingly, we pursue the following performance comparisons.

1) Time reversal gain over conventional detection-Target channel response known: we compare the TRCMF with the CDCMF.

2) Time reversal gain over conventional detection-Target channel response unknown: we compare the TR-GLRT with the ED.

3) Performance loss due to lack of knowledge of the target channel response: We compare the TRCMF with the TR-GLRT and the CDCMF with the ED.

The test statistics for the four detectors were derived in Section III. In that section, we also studied analytically the performance of the three detectors-the CDCMF, ED, and TRCMF-deriving analytical expressions for the probabilities of false alarm $P_{\mathrm{FA}}$ and detection $P_{\mathrm{D}}$, as well as the thresholds $\eta$, in terms of either the error function or the cumulative distribution function of $\chi$-square variables. For the TR-GLRT, we cannot derive these analytical expressions. We study its performance experimentally.

We start by describing the two experimental setups used to collect the electromagnetic data: channel I is propagation in free space in a cluttered environment and channel II is propagation in a duct. We detail each of these.

Channel I-Free Space Propagation in Cluttered Environment: The experimental setup is in Fig. 1. The time-domain waveform is produced by stepped frequency synthesis. The transmitted signal has $2 \mathrm{GHz}$ bandwidth with center frequency at $5 \mathrm{GHz}$, which corresponds to a wavelength $\lambda_{\mathrm{c}}=6 \mathrm{~cm}$. This signal is generated with an Agilent 89610A block vector network analyzer (VNA) in Fig. 1. We capture both the in-phase (I channel) and quadrature ( $Q$ channel) streams of the impulse response. The transmitter and receiver antennas are two horn 


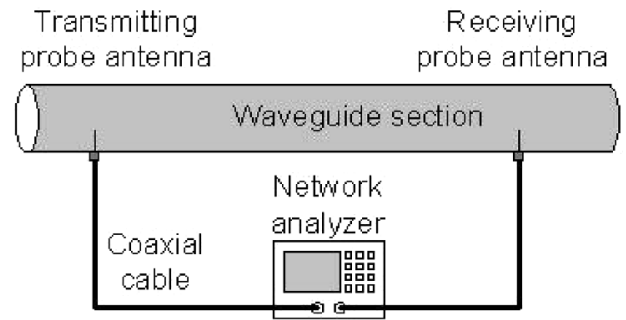

Fig. 2. Channel II: Duct. Operating frequency range is $2-3 \mathrm{GHz}$. Three-meter metal pipe duct with diameter of $0.3 \mathrm{~m}$ with metal caps. Transmitting and receiving antennas are monopole probes.

antennas, indicated by the letters $\mathrm{A}$ and $\mathrm{B}$ in the figure, both with operational bandwidths from 4 to $6 \mathrm{GHz}$. These two antennas are mounted in a slider that moves in rails as shown in the figure, with their positions computer controlled; Fig. 1 shows in dark and light gray two different possible positions for the antennas $\mathrm{A}$ and $\mathrm{B}$. The baseline separating these antennas can be up to $2 \mathrm{~m}$ (roughly $33 \lambda_{\mathrm{c}}$ ), as shown in the figure. The total $2 \mathrm{GHz}$ bandwidth is divided evenly into $Q-1=200$ bins. The radiated signal is scattered by 20 scatterers, shown as circles in Fig. 1. The scatterers are a mixture of copper pipes and solid dielectric pipes with $1.3 \mathrm{~cm}$ diameter and $3.2 \mathrm{~cm}$ outer diameter, respectively. The scatterers are placed in front of an absorbing wall that is $2.6 \mathrm{~m}$ (roughly $43 \lambda_{c}$ ) away from the antennas. A target, represented as a triangle, is immersed in the cloud of scatterers. The target is simply an additional copper pipe of the same $1.3 \mathrm{~cm}$ diameter. The impulse response $h_{c}(t)$ of the scattering environment of Channel I is the top plot in Fig. 3. The observation time window length is $100 \mathrm{~ns}$.

Channel II-Duct: The experimental setup is shown in Fig. 2. Again, the stepped frequency synthesis is performed to produce the time domain signal. The signal is transmitted through a $3 \mathrm{~m}$ metal pipe with metal caps. The diameter of the duct is $30.5 \mathrm{~cm}$. It operates like a resonant cavity, with a rich scattering environment. The transmitting and receiving antennas are monopole probes. The transmitted signal has $1 \mathrm{GHz}$ bandwidth with center frequency at $2.5 \mathrm{GHz}$, which corresponds to a wavelength $\lambda_{c}=12 \mathrm{~cm}$. This signal is generated with the same block VNA as with the channel I. We capture both the in-phase (I channel) and quadrature ( $Q$ channel) streams of the impulse response. The total $1 \mathrm{GHz}$ bandwidth is divided evenly into $Q-1=200$ bins. The impulse response $h_{c}(t)$ is the bottom plot in Fig. 3. The observation time window length is $200 \mathrm{~ns}$.

We first study the type of each of these two channels and compute the corresponding SNRG given by (85). The plot at the top of Fig. 4 shows the magnitude and phase of the target channel response $H_{t}\left(\omega_{q}\right)$ for channel I, while the plot in the middle shows its type or empirical distribution. The plots in Fig. 5 show the corresponding results for the channel II. Note the longer, heavier tail of the type of channel II. Although the number of frequency bins is for both channels $Q-1=200$, we compute the SNRG (85), or the ratio $\gamma$ in (90), with only the 40 equally spaced bins that are used below in studying the performance of the four detectors. We obtain $\mathrm{SNRG}_{\mathrm{I}}=2.36 \mathrm{~dB}$ and $\mathrm{SNRG}_{\mathrm{II}}=9.05 \mathrm{~dB}$, respectively. These gains show that the richer the scattering environment is the larger the gains to be expected.
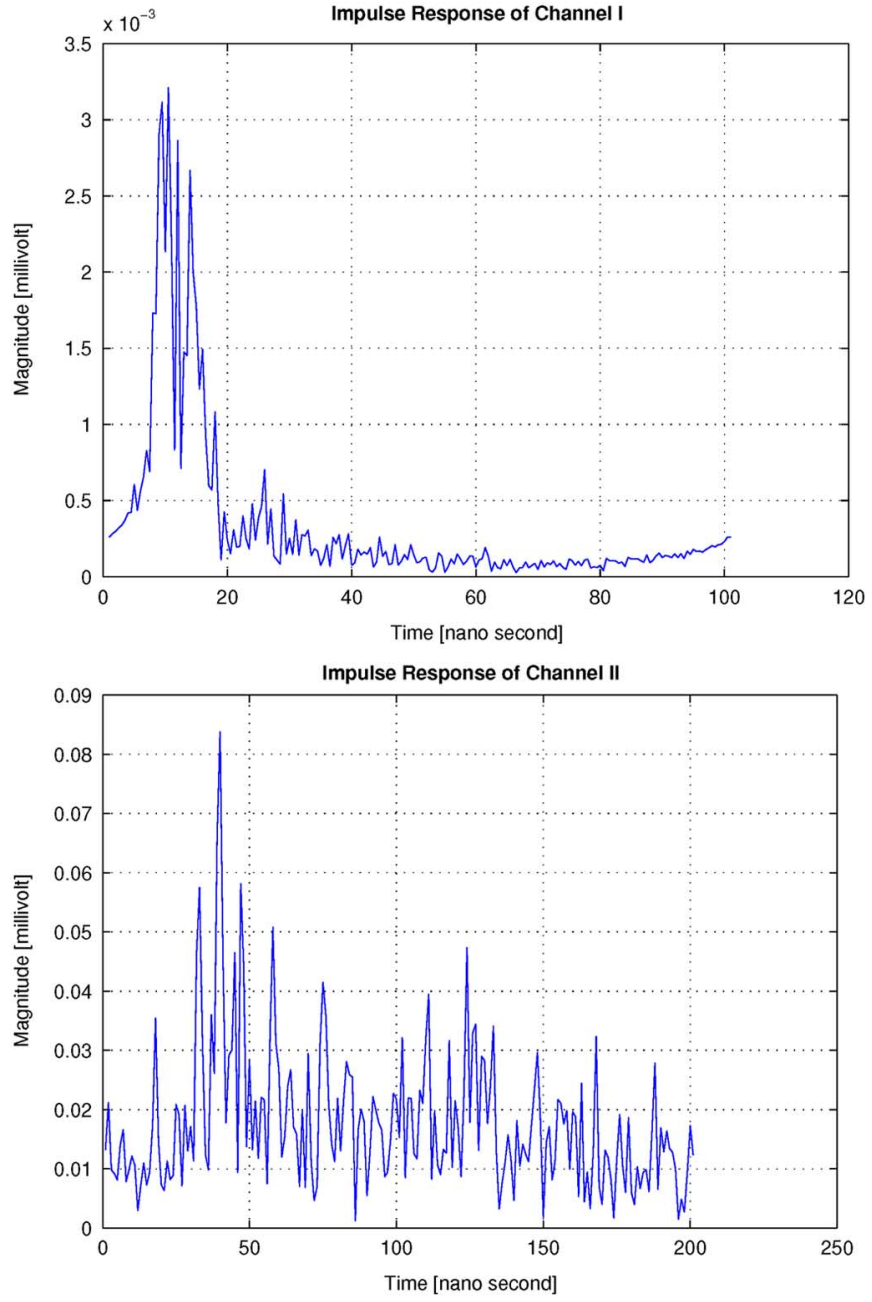

Fig. 3. (Top) Impulse response $h_{c}(t)$ of Channel I: free space propagation scattering environment. (Bottom) Impulse response $h_{c}(t)$ of Channel II: duct. Both time-domain impulse responses are obtained by the inverse fast Fourier transform of the frequency measurements, i.e., stepped frequency synthesis. Channel I is measured between $4-6 \mathrm{GHz}$ with center frequency of $5 \mathrm{GHz}$; Channel II is measured between $2-3 \mathrm{GHz}$ with center frequency of $2.5 \mathrm{GHz}$.

We now study experimentally the error performance of the four detectors. We follow the setup explained in Section II where we first learn the clutter frequency response $H_{c}\left(\omega_{q}\right)$ when no target is present and then use background subtraction to suppress the clutter. This leads to the conventional detection problem defined by (41) and to the time reversal detection problem defined by (76) in Section III. To study performance, we plot the probability of detection $P_{\mathrm{D}}$ as a function of the SNR for a fixed probability of false alarm $P_{\mathrm{FA}}$. To obtain noisy backscatterer at different SNR, we add numerically generated zero-mean white Gaussian noise to the real data EM backscatter. The SNR is defined by

$$
\mathrm{SNR}=\frac{E\left\{\left\|\mathbf{S h}_{t}\right\|^{2}\right\}}{E\left\{\|\mathbf{w}\|^{2}\right\}}=\frac{\sum_{q=0}^{Q-1}\left|S\left(\omega_{q}\right) H_{t}\left(\omega_{q}\right)\right|^{2}}{Q \sigma_{w}^{2}}
$$

This noise is background noise. Through the experiments, we set $S\left(\omega_{q}\right)=1, q=0, \ldots, Q-1$, and $\sigma_{v}^{2}=\sigma_{w}^{2}=1$. 

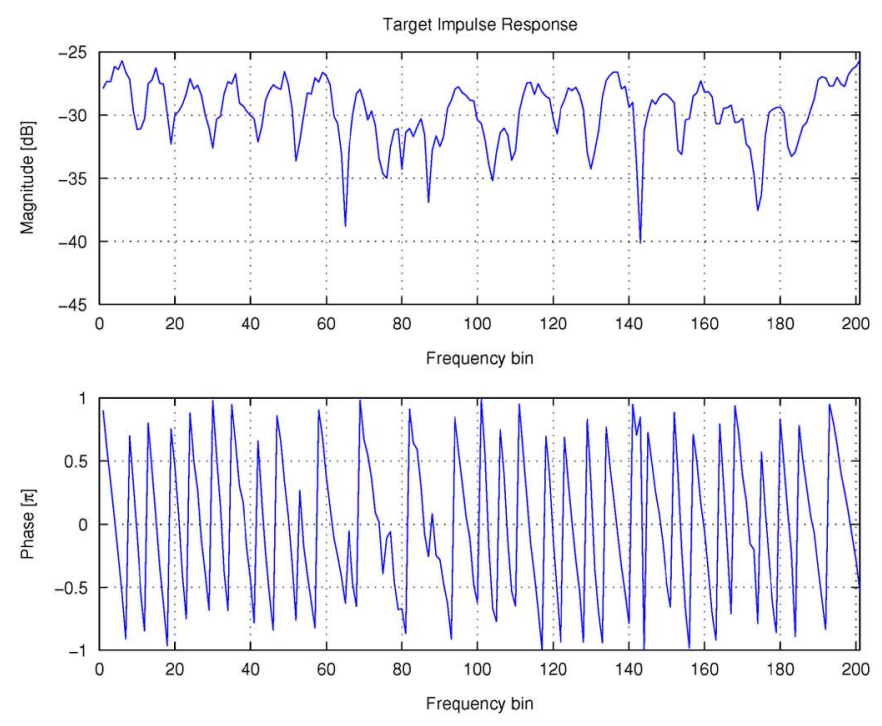

Histogram of Channel I

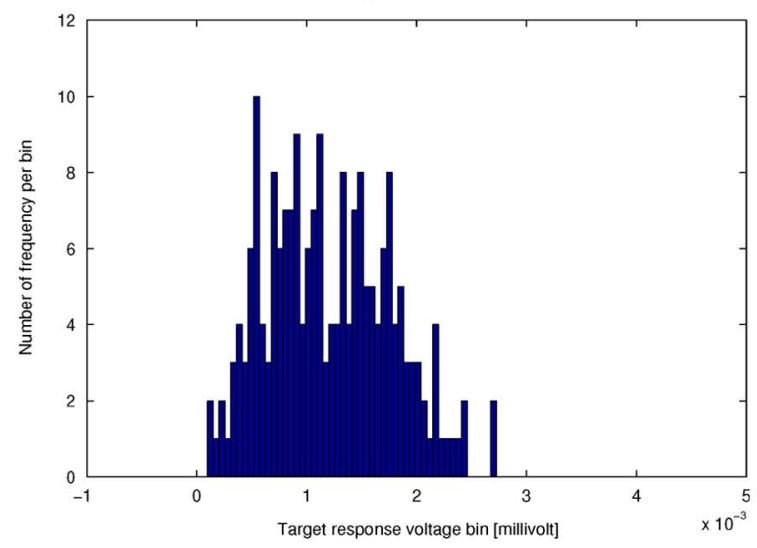

Fig. 4. Channel I: (Top) $\left|H_{t}\left(\omega_{q}\right)\right|$ and phase of $H_{t}\left(\omega_{q}\right)$. (Bottom) Type or empirical distribution of target channel response $H_{t}\left(\omega_{q}\right)$.

The total signal energy $(1 / Q)\left\|\mathbf{S h}_{t}\right\|^{2}$ is scaled to meet different SNR levels.

We determine the threshold $\eta$, the $P_{\mathrm{FA}}$, and the $P_{\mathrm{D}}$ by Monte Carlo for the TR-GLRT when we fix the $P_{\mathrm{FA}}=10^{-2}$. We generated 8000 independent trials and computed the test statistic given by (81), using the ML-estimate for the target channel response in (84). The resulting 8000 test statistics are sorted in ascending order. The threshold is then selected to result in a $P_{\mathrm{FA}}=10^{-2}$. Once the threshold is chosen, to compute the $P_{\mathrm{D}}$, we generate 8000 new independent data snapshots containing both target and noise. We then compute the test statistic and compare it with the corresponding threshold. The percentage of the number of times that the test statistic exceeds the threshold when the target is present is counted as the detection probability $P_{\mathrm{D}}$.

For the other three detectors CDCMF, TRCMF, and ED, the thresholds and the $P_{\mathrm{D}}$ at fixed $P_{\mathrm{FA}}$ can be determined analytically with the expressions provided in Section III. To confirm the validity of the experiments, we used the same procedure and the same 8000 independent trials to compute the thresholds $\eta$ and the probabilities $P_{\mathrm{D}}$ and $P_{\mathrm{FA}}$ for each of these detectors. We repeated the study for a different value of the false alarm
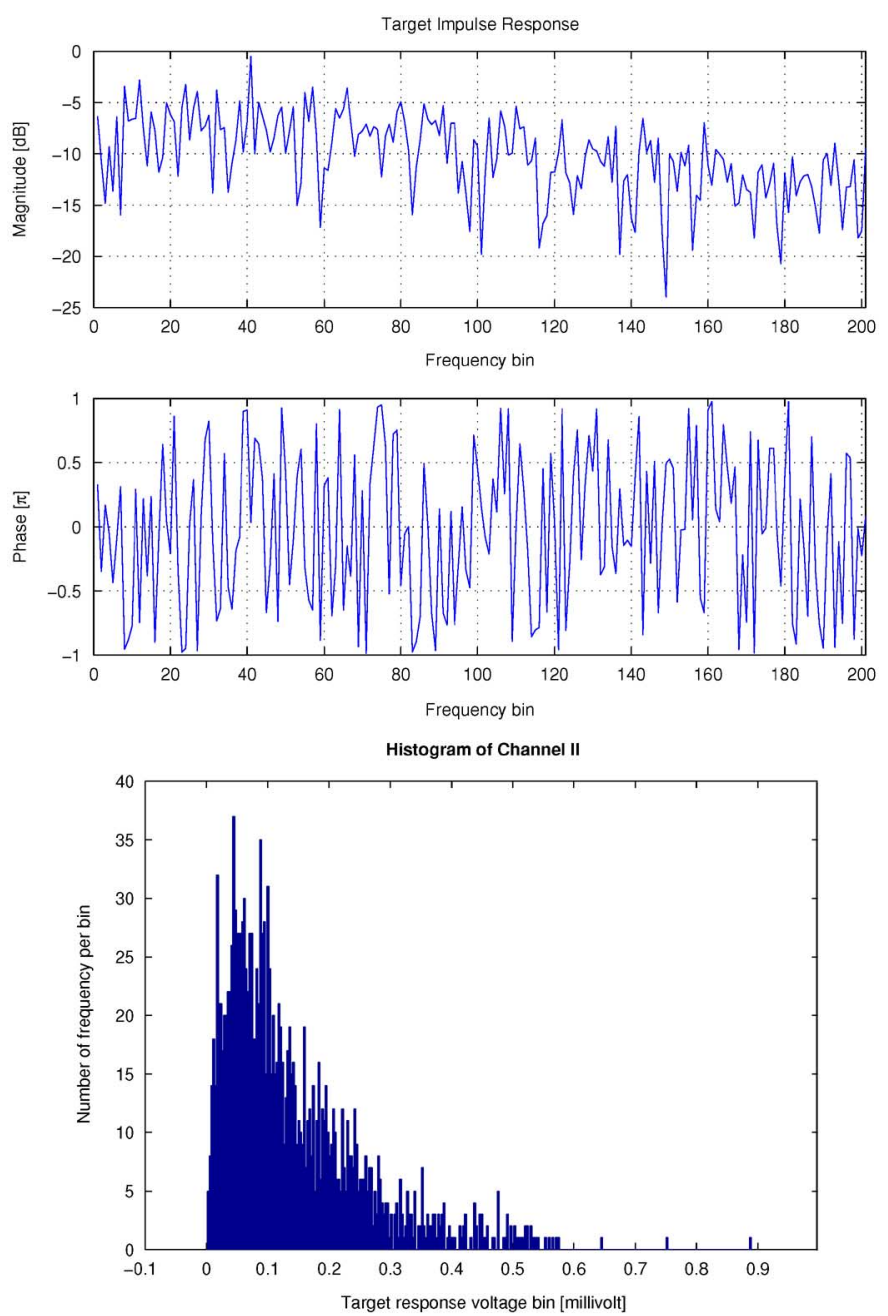

Fig. 5. Channel II: (Top) $\left|H_{t}\left(\omega_{q}\right)\right|$ and phase of $H_{t}\left(\omega_{q}\right)$. (Bottom) Type or empirical distribution of target channel response $H_{t}\left(\omega_{q}\right)$.

probability, namely, $P_{\mathrm{FA}}=10^{-3}$, with 40000 Monte Carlo independent runs.

Figs. 6 and 7 show, for $P_{\mathrm{FA}}=10^{-2}$ and $P_{\mathrm{FA}}=10^{-3}$, the analytical and experimental results for channel I, with target channel response in Fig. 4, for the four detectors CDCMF, TRCMF, ED, and TR-GLRT. The analytical results correspond to the plots labeled with the prefix "Ana." In Figs. 8 and 9, we show the corresponding experimental results for channel B, i.e., the duct channel, whose type is shown in Fig. 5.

We make a few comments. First, we note that there is very good agreement between the experimental results and the theoretical performance predictions in Section III for the CDCMF, TRCMF, and ED detectors; this gives a good indication that the number of independent snapshots used to determine the thresholds and the error probabilities is statistically significant.

A second comment is with respect to the detection gain SNRG provided by time reversal over conventional detection. From the plots, we see that SNRG for channel I is about $2.4 \mathrm{~dB}$ and about $9 \mathrm{~dB}$ for channel II, in agreement with the theoretical predictions computed from the channel type plots in Figs. 4 and 5 , respectively. 

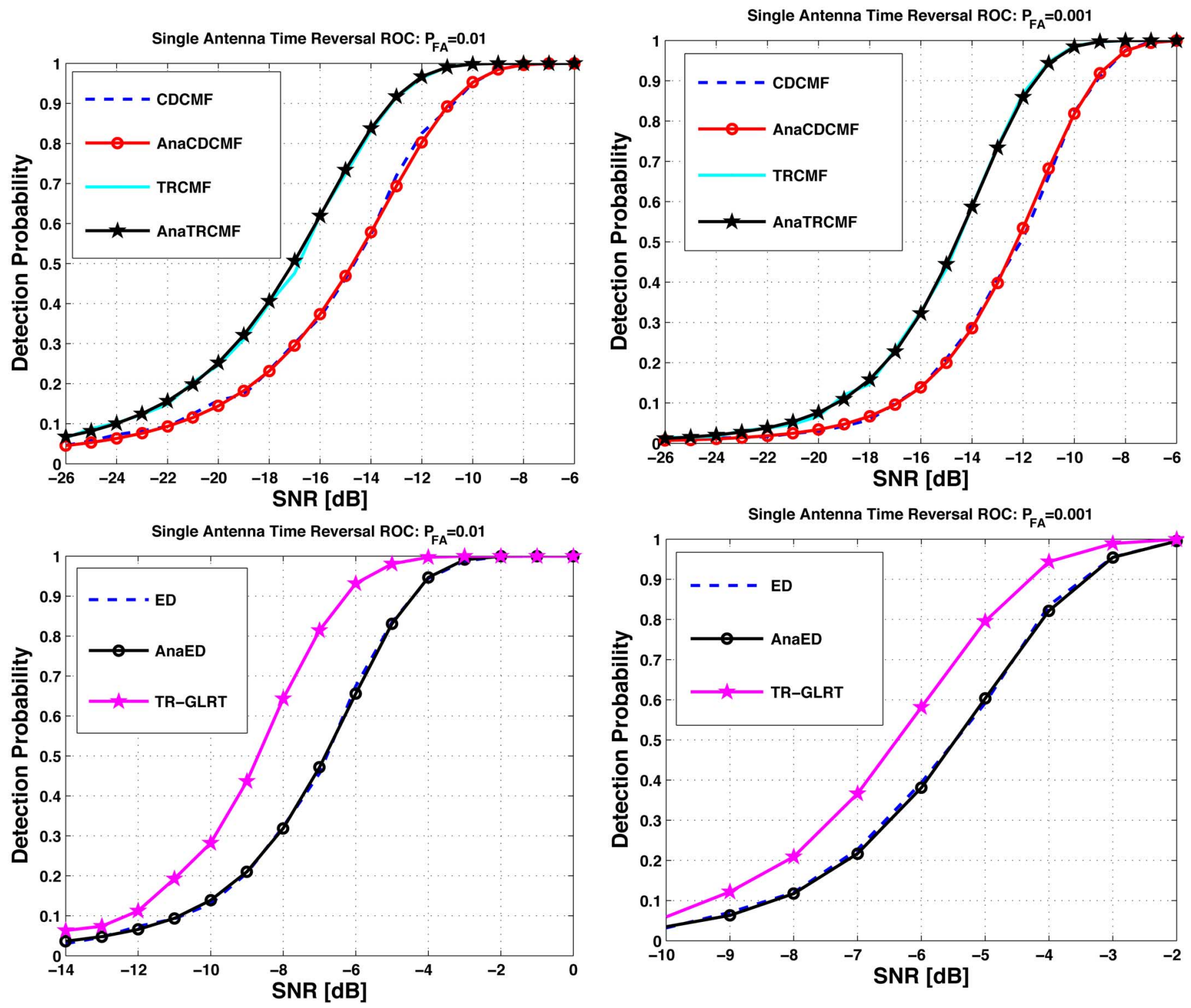

Fig. 6. Detection probability versus SNR for CDCMF, TRCMF, ED, and TR-GLRT for channel I (Fig. 4). False alarm rate $P_{\mathrm{FA}}=10^{-2}$. The total number of data snapshots is two: for CDCMF and ED, $M_{Y}=2$; for TRCMF, $M_{X}=2 ;$ and for TR-GLRT, $M_{X}=1$ and $M_{Y}=1$.

When the target channel response is not known and we use the generalized likelihood ratio tests, the time reversal gain is about $1 \mathrm{~dB}$ for channel I and $2 \mathrm{~dB}$ for channel II. Also, the performance loss when the target channel response is not known with respect to when it is known can be significant. For instance, there is about a $9 \mathrm{~dB}$ loss at the target detection probability of $P_{D}=0.5$ shown in Fig. 6. This loss can be mitigated if more snapshots are available. Further, note that even for the same total number of snapshots, the performance gain provided by time reversal over conventional detection can increase significantly if, as noted in the Remark in Section II-B, the number of snapshots $M_{X}$ of the time reversed signal is increased while the number of snapshots of the direct signal $M_{Y}$ is decreased. Thus we keep $M_{X}+M_{Y}=$ $2 M$. In the limit, we can set $M_{X}=2 M-1$ and $M_{Y}=1$.

Fig. 7. Probability versus SNR for CDCMF, TRCMF, ED, and TR-GLRT for channel I (Fig. 4). False alarm rate $P_{\mathrm{FA}}=10^{-3}$. The total number of data snapshots is two: for CDCMF and ED, $M_{Y}=2$; for TRCMF, $M_{X}=2$; and for TR-GLRT, $M_{X}=1$ and $M_{Y}=1$.

\section{SUMMARY}

This paper studies the question of how much detection gain time reversal provides over conventional detection. For each of these two approaches to the target in clutter binary hypothesis testing, we consider two scenarios: ideal detection, where we assume known the target channel frequency response $H_{t}\left(\omega_{q}\right)$, and realistic, where $H_{t}\left(\omega_{q}\right)$ is assumed unknown. We derive the corresponding test statistics in Section III: the conventional detection channel matched filter, the time reversal channel matched filter, the energy detector, which is the generalized likelihood ratio test for the realistic conventional detection problem, and the time reversal generalized likelihood ratio test for the realistic time reversal detection problem. For the first three detectors, we derive analytical expressions for 

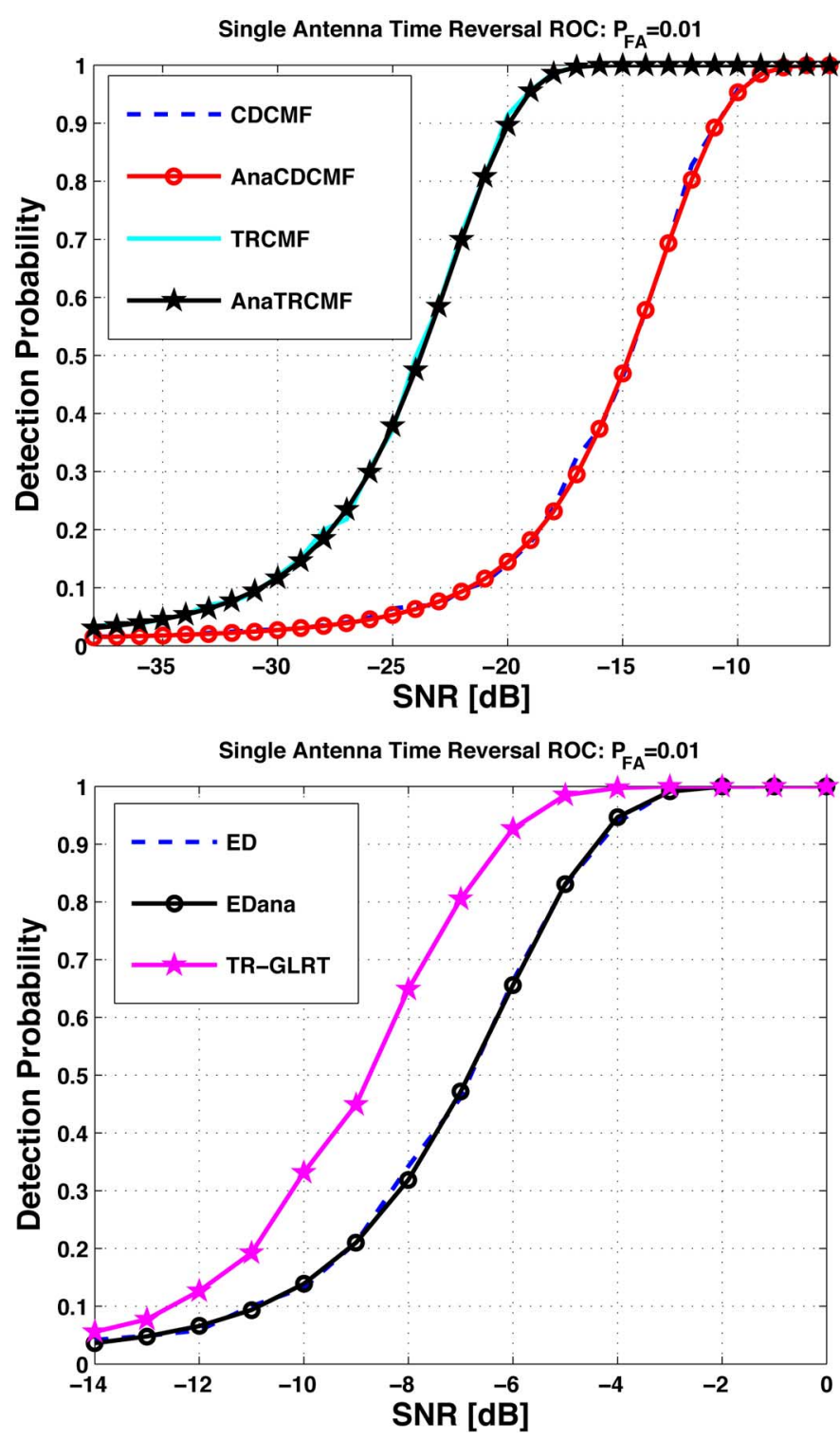

Fig. 8. Detection probability versus SNR for CDCMF, TRCMF, ED, and TR-GLRT for channel II (Fig. 2). False alarm rate $P_{\mathrm{FA}}=10^{-2}$. The total number of data snapshots is two: for CDCMF and ED, $M_{Y}=2$; for TRCMF, $M_{X}=2 ;$ and for TR-GLRT, $M_{X}=1$ and $M_{Y}=1$.

the threshold and for the error probabilities. Finally, we test all four detectors with real electromagnetic data collected in the laboratory for two channels-free space cluttered environment channel and a duct channel.

The analysis and experiments show that time reversal can provide significant detection gains and that these gains are directly related to how rich the target channel response is: channels where the clutter induces a richer target channel frequency response will lead to larger gains for time reversal detection over conventional detection. Time reversal provides a simple methodology to adapt the transmitted waveform to the channel. It is this automatic adaptation that explains the detection gains.
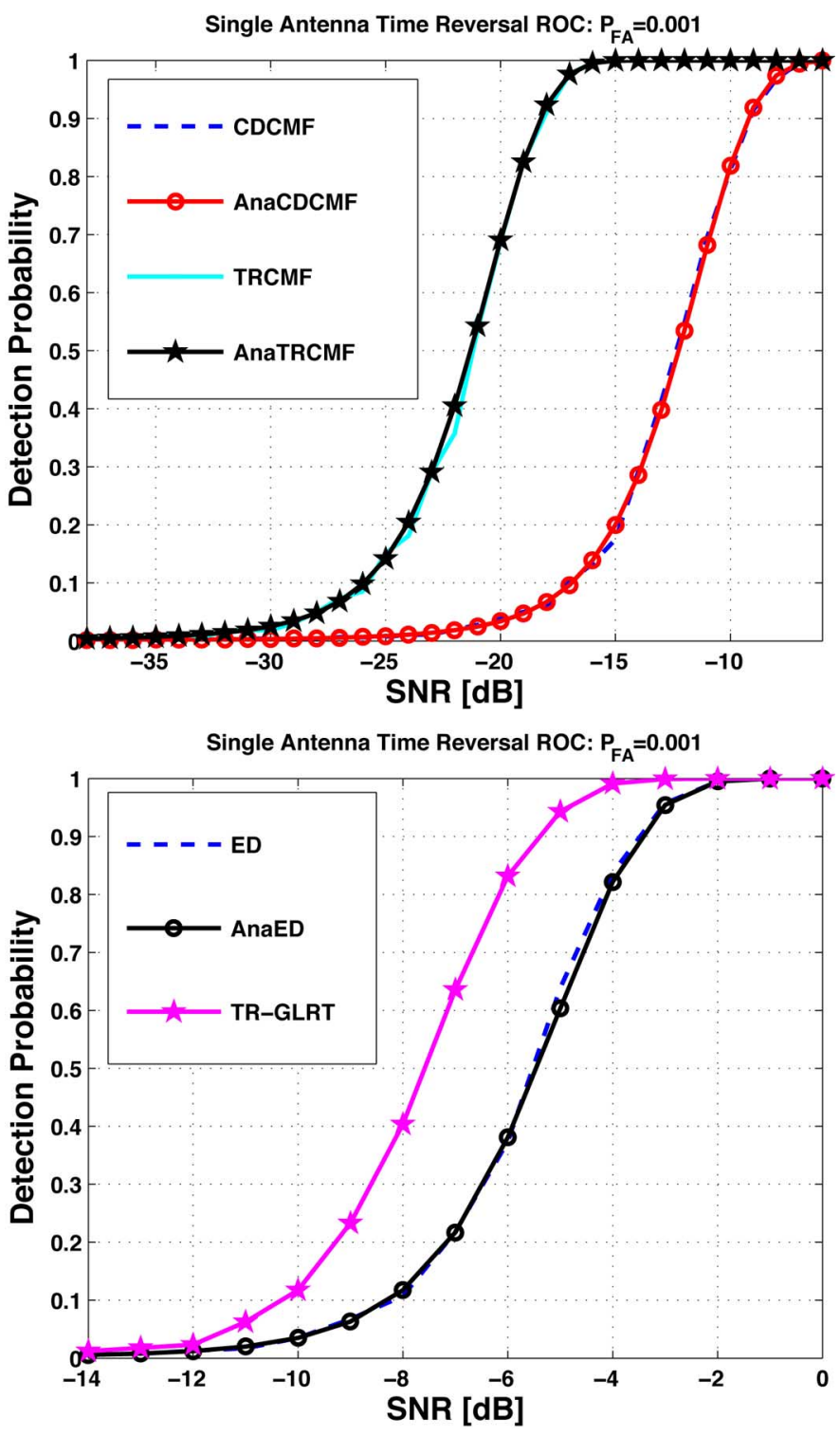

Fig. 9. Detection probability versus SNR for CDCMF, TRCMF, ED, and TR-GLRT for channel II (Fig. 2). False alarm rate $P_{\mathrm{FA}}=10^{-3}$. The total number of data snapshots is two: for CDCMF and ED, $M_{Y}=2$; for TRCMF, $M_{X}=2$; and for TR-GLRT, $M_{X}=1$ and $M_{Y}=1$.

A more comprehensive experimental study comparing time reversal detection with matched filter detection is carried out in [25].

\section{ACKNOWLEDGMENT}

The authors thank Prof. D. Stancil, Prof. J. Zhu, A. Cepni, Y. Jiang and B. Henty for the discussions held and for providing the electromagnetic data for channels I and II.

\section{REFERENCES}

[1] T. M. Cover and J. A. Thomas, Elements of Information Theory. New York: Wiley, 1991

[2] M. Fink, C. Prada, F. Wu, and D. Cassereau, "Self focusing in inhomogeneous media with time reversal acoustic mirrors," in IEEE Ultrasonics Symp., Montreal, PQ, Canada, 1989, vol. 1, pp. 681-686. 
[3] C. Prada, F. Wu, and M. Fink, "The iterative time reversal mirror: A solution to self-focusing in the pulse echo mode," J. Acoust. Soc. Amer., vol. 90, pp. 1119-1129, 1991.

[4] M. Fink, "Time reversal of ultrasonic fields. Part I: Basic principles," IEEE Trans. Ultrason., Ferroelectron., Frequency Contr., vol. 39, pp. 555-566, Sep. 1992.

[5] C. Dorme and M. Fink, "Focusing in transmit-receive mode through inhomogeneous media: The time reversal matched filter approach," $J$. Acoust. Soc. Amer., vol. 98, no. 2, pt. 1, pp. 1155-1162, Aug. 1995.

[6] M. Fink, "Time reversed acoustics," Phys. Today, vol. 50, no. 3, pp. 34-40, 1997.

[7] W. A. Kuperman, W. S. Hodgkiss, and H. C. Song, "Phase conjugation in the ocean: Experimental demonstration of an acoustic time-reversal mirror," J. Acoust. Soc. Amer., vol. 103, no. 1, pp. 25-40, Jan. 1998.

[8] H. C. Song, W. A. Kuperman, W. S. Hodgkiss, T. Akal, and C. Ferla, "Iterative time reversal in the ocean," J. Acoust. Soc. Amer., vol. 105, no. 6, pp. 3176-3184, Jun. 1999.

[9] L. Borcea, G. Papanicolaou, C. Tsogka, and J. Berryman, "Imaging and time reversal in random media," Inverse Problems, vol. 18, pp. 1247-1289, 2002

[10] S. K. Lehman and A. J. Devaney, "Transmission mode time-reversal super-resolution imaging," J. Acoust. Soc. Amer., vol. 113, no. 5, pp. 2742-2753, May 2003.

[11] A. J. Devaney, "Time reversal imaging of obscured targets from multistatic data," IEEE. Trans. Antennas Propag., vol. 53, pp. 1600-1610, May 2005

[12] J. V. Candy, A. W. Meyer, A. J. Poggio, and B. L. Guidry, "Timereversal processing for an acoustic communications experiment in a highly reverberant environment," J. Acoust. Soc. Amer., vol. 115, no. 4, pp. 1621-1631, Apr. 2004

[13] D. Flore and E. Lindskog, "Time-reversal space-time block coding vs. transmit diversity-A comparison based on a GSM-like system," in DSP'2000 9th Digital Signal Process. Workshop, TX, Oct. 2000, pp. 1-5 [Online]. Available: http://spib.ece.rice.edu/DSP2000/program, Waldemar Ranch Resort.

[14] C. Oestges, A. D. Kim, G. Papanicolaou, and A. J. Paulraj, "Characterization of space time focusing in time reversed random fields," IEEE Trans. Antennas Propag., vol. 53, pp. 283-293, Jan. 2005.

[15] B. E. Henty and D. D. Stancil, "Multipath enabled super-resolution for RF/microwave communication using phase-conjugate arrays," Phys. Rev. Lett., vol. 93, no. 24, p. 243904, Dec. 2004.

[16] G. Lerosey, J. de Rosny, A. Tourin, A. Derode, G. Montaldo, and M. Fink, "Time reversal of electromagnetic waves," Phys. Rev. Lett., vol. 92, p. 194301, May 2004

[17] J. M. F. Moura, Y. Jin, D. Stancil, J. Zhu, A. Cepni, Y. Jiang, and B. Henty, "Single antenna time reversal adaptive interference cancelation," in IEEE Int. Conf. Signal Process. (ICASSP'05), Philadelphia, PA, Mar. 2005, vol. IV, pp. 1121-1124, IEEE.

[18] T. W. Anderson, An Introduction to Multivariate Statistical Analysis, 2nd ed. New York: Wiley, 1971.

[19] A. V. Oppenheim, A. S. Willsky, and S. H. Nawab, Signals and Systems, 2nd ed. Upper Saddle River, NJ: Prentice-Hall, 1996.

[20] E. A. Lee and D. G. Messerschmitt, Digital Communication, 2nd ed. Norwell, MA: Kluwer Academic, 1997.

[21] H. L. VanTrees, Detection, Estimation, and Modulation Theory: Part I. New York: Wiley, 1968.

[22] S. M. Kay, Detection Theory, Fundamentals of Statistical Signal Processing. Upper Saddle River, NJ: Prentice-Hall, 1998, vol. 2, “,”, vol. , pp. - , .

[23] N. L. Johnson and S. Kotz, Continuous Univariate Distributions, 2nd ed. New York: Wiley, 1970, ch. 28.

[24] M. H. Hayes, Statistical Digital Signal Processing and Modeling. New York: Wiley, 1996.
[25] J. M. F. Moura, Y. Jin, D. Stancil, J. Zhu, A. Cepni, Y. Jiang, and B. Henty, "Time reversal detection and matched filter in the electromagnetic domain,", unpublished.

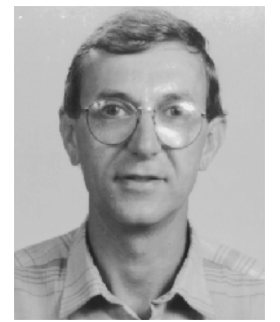

José M. F. Moura (S'71-M'75-SM'90-F'94) received the engenheiro electrotécnico degree from Instituto Superior Técnico (IST), Lisbon, Portugal, in 1969 and the M.Sc. and E.E. degrees in 1973 and D.Sc. degrees in electrical engineering and computer science in 1975, all from the Massachusetts Institute of Technology (MIT), Cambridge.

$\mathrm{He}$ is a Professor of electrical and computer engineering and of BioMedical Engineering at Carnegie-Mellon University, Pittsburgh, PA, where he is a Founding Codirector of the Center for Sensed Critical Infrastructures Research. In the academic year 2006-2007, he is a visiting Professor at MIT. He was on the Faculty of IST (1975-84) and has held Visiting Faculty appointments at MIT (1984-1986 and 1999-2000) and as a Research Scholar at the University of Southern California (summers of 1978-1981). His research interests include statistical and algebraic signal, image and bioimaging, video processing, and digital communications. He has published more than 300 technical journal and conference papers, is the coeditor of two books, has received six U.S. patents on image and video processing and digital communications, and has given numerous invited seminars at US and European universities and industrial and government laboratories.

Dr. Moura is a Fellow of the American Association for the Advancement of Science and a Corresponding Member of the Academy of Sciences of Portugal (Section of Sciences). He is a member of Sigma Xi, AMS, IMS, and SIAM. He received the 2003 IEEE Signal Processing Society Meritorious Service Award and in 2000 the IEEE Millenium medal. Dr. Moura has served the IEEE Signal Processing Society (SPS) in several capacities, including President Elect (2006-2007), Vice-President for Publications and member of the Board of Governors (2000-2002), Editor in Chief for the IEEE TRANSACTIONS ON SigNAL PROCESSING (1975-1999), interim Editor in Chief for the IEEE SIGNAL PROCESSING LETTERS (December 2001-May 2002), Founding Member of the Bioimaging and Signal Processing (BISP) Technical Committee, and member of several other technical committees. He was Vice-President for Publications for the IEEE Sensors Council (2000-2002) and is or was on the Editorial Board of several journals, including the PROCEEDINGS OF THE IEEE, the IEEE SIGNAL PROCESSING MAgAZINE, and the ACM Transactions on Sensor Networks. He chaired the IEEE TAB Transactions Committee (2002-2003) that joins the more than 80 Editors in Chief of the IEEE TRANSACTIONS and served on the IEEE TAB Periodicals Review Committee (2002-2005). He is or was on the Steering Committees of the International Symposium on BioImaging and of the International Conference on Information Processing and Sensor Networks, and has been on the Program Committee of more than 30 conferences and workshops. He was on the IEEE Press Board (1991-1995).

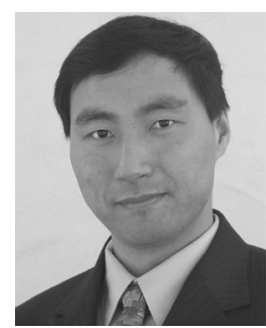

Yuanwei Jin (S'99-M'04) received the B.S. and M.S. degrees from East China Normal University, Shanghai, in 1993 and 1996, respectively, and the $\mathrm{Ph} . \mathrm{D}$. degree in electrical engineering from the University of California at Davis in 2003.

From 2003 to 2004, he was a Visiting Researcher with the University of California at Santa Cruz. Since 2004, he has been a Postdoctoral Research Fellow with Carnegie-Mellon University, Pittsburgh, PA. His research interests are in the general area of statistical signal processing, including estimation and detection theory, higher order statistics, equalization, adaptive filtering, and sensor array processing with applications in sonar/radar and wireless communications systems.

Dr. Jin received the Earle C. Anthony Fellowship from the University of California at Davis. 